\title{
Campbell penetration depth in Fe-based superconductors
}

by

\section{Plengchart Prommapan}

\author{
A thesis submitted to the graduate faculty \\ in partial fulfillment of the requirements for the degree of \\ MASTER OF SCIENCE
}

Major: Condensed Matter Physics

Program of Study Committee:

Ruslan Prozorov, Major Professor

Sergey Bud'ko

John Lajoie

R. William McCallum

lowa State University

Ames, lowa

2011 


\section{TABLE OF CONTENTS}

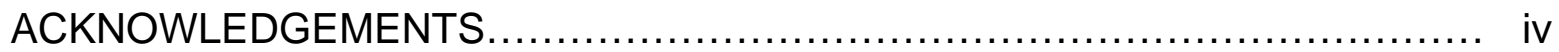

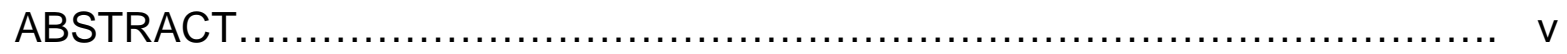

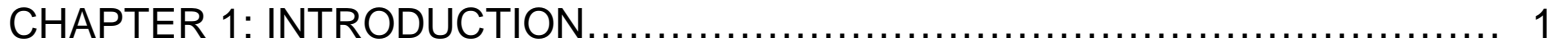

CHAPTER 2: LABUSCH PARAMETER AND

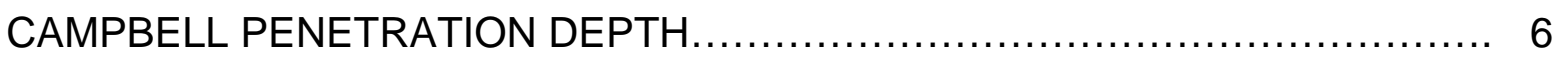

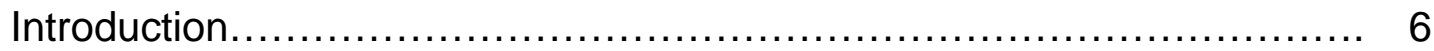

Labusch parameter............................................... 7

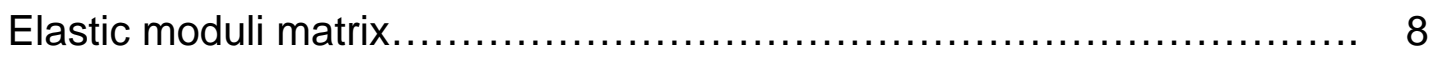

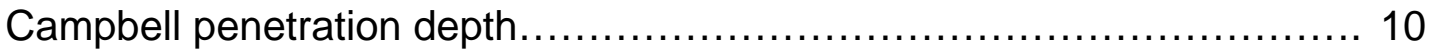

Critical current from Campbell penetration depth..................... 13

Dependency of current in Campbell penetration depth.................. 14

CHAPTER 3: TUNNEL-DIODE RESONATOR $\ldots \ldots \ldots \ldots \ldots \ldots \ldots \ldots \ldots \ldots \ldots \ldots \ldots \ldots \ldots$

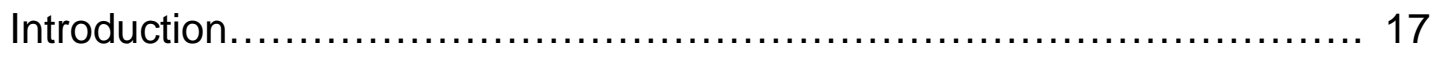

TDR circuit component........................................... 18

Magnetic penetration depth measurement.......................... 21

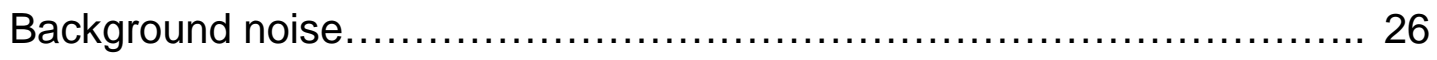

CHAPTER 4: CAMPBELL PENETRATION DEPHT IN

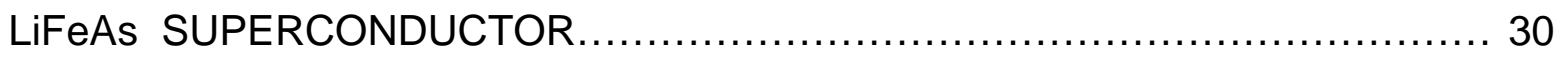




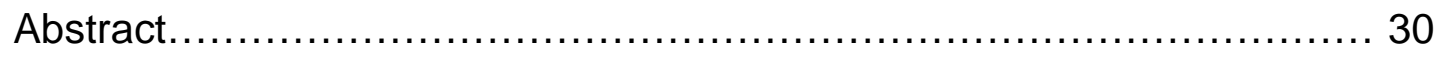

Sample and method...................................................... 31

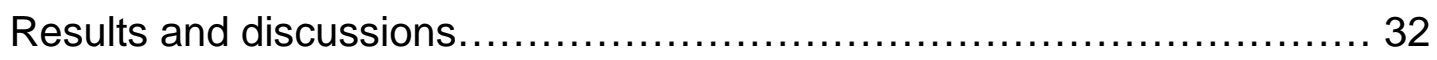

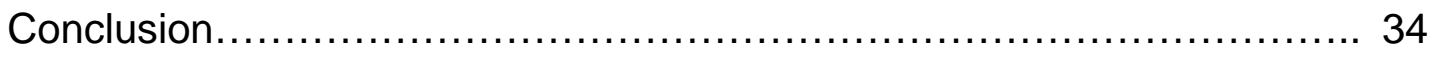

CHAPTER 5: CAMPBELL PENETRATION DEPHT IN

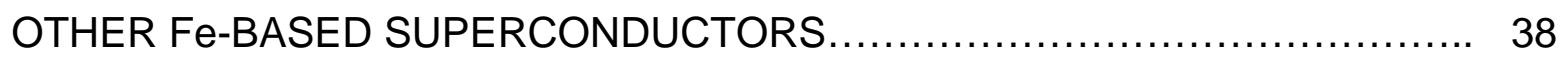

Non-irradiated FeNi-122 ............................................ 39

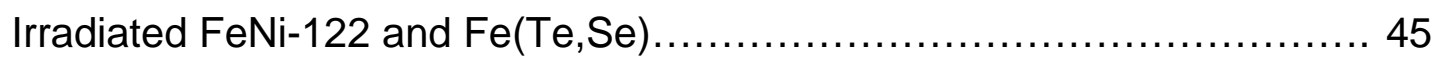

BaK-122 and BaP-122 .............................................. 49

CHAPTER 6: CAMPBELL PENETRATION DEPHT IN

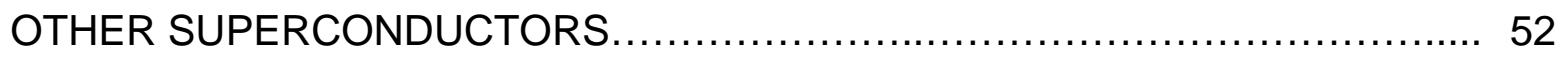

High purity niobium foil............................................... 52

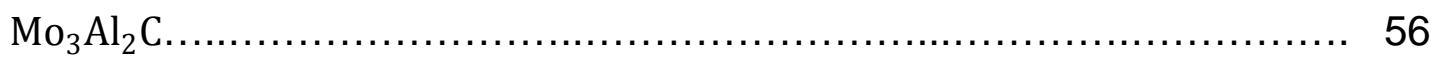

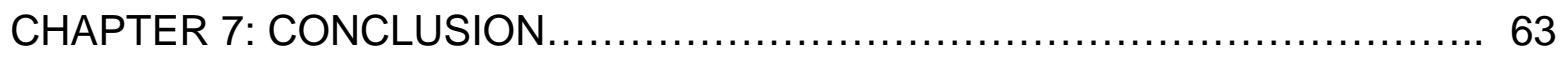

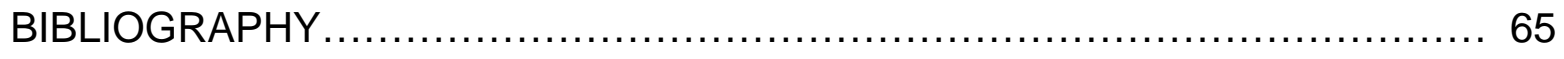




\section{Acknowledgement}

I would like to take this opportunity to thank the people who have provided help and support in the various aspects of my life during these past years. First and foremost, I would like to thank my major professor, Professor Ruslan Prozorov for his valuable guidance and support. Without his endless encouragements, I would have never made it this far in my academic life. I thank the member of my committee, Professor Sergey Bud'ko, Professor John Lajoie, and Professor R. William McCallum for their time and contribution to this work. I would also like to thank Makariy Tanatar for all his valuable support and advice in the laboratory. I would like to thank all the past and present members of the Prozorov group; working alongside them has been very enjoyable and beneficial to me. I would additionally like to thank all of my friends for helping me enjoy life in Ames. In particular, I would like to thank Steven Yeninas, Dajing Wu, Chorthip Peeraphatdit, Nattapol Ploymaklam, and Erick Blomberg for all their help in writing this thesis. Finally, I would like to thank my family for their warm support and unwavering encouragement. I really appreciate it. 


\begin{abstract}
A "true" critical current density, $j_{c}$, as opposite to commonly measured relaxed persistent (Bean) current, $j_{B}$, was extracted from the Campbell penetration depth, $\lambda_{C}(T, H)$ measured in single crystals of LiFeAs, and optimally electron-doped $\mathrm{Ba}\left(\mathrm{Fe}_{0.954} \mathrm{Ni}_{0.046}\right)_{2} \mathrm{As}_{2}$ (FeNi122). In LiFeAs, the effective pinning potential is nonparabolic, which follows from the magnetic field - dependent Labusch parameter $\alpha$. At the equilibrium (upon field - cooling), $\alpha(H)$ is non-monotonic, but it is monotonic at a finite gradient of the vortex density. This behavior leads to a faster magnetic relaxation at the lower fields and provides a natural dynamic explanation for the fishtail (second peak) effect. We also find the evidence for strong pinning at the lower fields. The inferred field dependence of the pinning potential is consistent with the evolution from strong pinning, through collective pinning, and eventually to a disordered vortex lattice. The value of $j_{c}(2 \mathrm{~K}) \simeq 1: 22 \times 10^{6} \frac{A}{\mathrm{~cm} 2}$ provide an upper estimate of the current carrying capability of LiFeAs. Overall, vortex behavior of almost isotropic, fully-gapped LiFeAs is very similar to highly anisotropic d-wave cuprate superconductors, the similarity that requires further studies in order to understand unconventional superconductivity in cuprates and pnictides.
\end{abstract}


In addition to LiFeAs, we also report the magnetic penetration depth in $\mathrm{BaFe}_{2} \mathrm{As}_{2}$ based superconductors including irradiation of FeNi122. In unirradiated FeNi122, the maximum critical current value is, $j_{c}(2 \mathrm{~K}) \simeq 3.3 \times 10^{6} \frac{\mathrm{A}}{\mathrm{cm}^{2}}$. The magnetic-dependent feature was observed near the transition temperature in $\mathrm{FeTe}_{0.53} \mathrm{Se}_{0.47}$ and irradiated FeNi122. Because of this feature, further studies are required in order to properly calibrate the Campbell penetration depth. Finally, we detected the crossing between the magnetic penetration depth and London penetration depth in optimally hold-doped $\mathrm{Ba}_{0.6} \mathrm{~K}_{0.4} \mathrm{Fe}_{2} \mathrm{As}_{2}$ (BaK122) and isovalent doped $\mathrm{BaFe}_{2}\left(\mathrm{As}_{0.7} \mathrm{P}_{0.3}\right)_{2}$ (BaP122). These phenomena probably coincide with anomalous Meissner effect reported in pnicitde superconductors [Prozorov et al. (2010b)] however more studies are needed in order to clarify this. 


\section{Chapter 1}

\section{Introduction}

The determination of the critical current density $j_{c}$ is one of the fundamental problems in the vortex physics of type-II superconductors. Not only it is important for the assessment of the current-carrying capabilities relevant for practical applications, but knowing the "true" $j_{c}$ is needed to understand microscopic mechanisms of vortex pinning. What is often called "critical current" is routinely determined from conventional DC magnetization measurements. Alas, this quantity is a convolution of "true" $j_{c}$ and magnetic relaxation during the characteristic time, $\Delta t$, of the experiment. For example, in the case of ubiquitous Quantum Design MPMS (SQUID) magnetometery, $\Delta t \geq 10 \mathrm{sec}$. We will call measured supercurrent $j_{B}$ to distinguish it from the "true" $j_{c}$ that is achieved when the vortices are de-pinned by the Lorentz force. By definition, $j_{c}$ is reached when the energy barrier for vortex motion vanishes, $U\left(j_{c}\right)=0$, whereas the measured current density $j_{B}$ is determined by $U\left(j_{B}\right)=$ $k B T \ln \left(1+\frac{\Delta t}{t_{0}}\right)$, where $t_{0} \lesssim 1 \mu \mathrm{sec}$ is the characteristic time scale that depends on both sample geometry and details of pinning [Geshkenbein and Larkin (1989); Vinokur, Feigel'man, and Geshkenbein (1991); Blatter et al. (1994); Yeshurun, Malozemoff, and Shaulov (1996); Burlachkov, Giller, and Prozorov (1998)]. This 
also results in a quite different temperature dependence of $j_{B}(T)$ compared to $j_{C}(T)$. Another approach to measure critical current density is to use AC susceptibility. Conventional time-domain susceptometers operate at frequencies $f \lesssim 10 \mathrm{kHz}$ (hence $\Delta t \approx 0.1 \mathrm{msec}$ ) and have large driving amplitudes, $H_{a c} \gtrsim 0.1$ Oe. Such perturbation displaces vortices from the potential wells and one can use harmonics analysis to determine frequency - dependent current density, $j_{B}(T, B, f)$. This technique has been applied in both global [Burlachkov, Giller, and Prozorov (1993)] and local [Prozorov et al. $(1994,1995)]$ forms.

In Fe-based superconductors, flux creep is substantial at all temperatures. Thus, measured $j_{B}$ is expected to be lower than $j_{c}$. Indeed, reports produce only moderate current densities, $j_{B} \lesssim 10^{6} \frac{A}{\mathrm{~cm}^{2}}$, unusual for low anisotropy high- $T_{c}$ materials [Prozorov et al. (2008,2009); Yang et al. (2008a,b); Kim et al. (2009); Shen et al. (2010); Pramanik et al. (2010)]. To illustrate, Figure 1-1 shows the relaxation of a magnetic moment at $15 \mathrm{~K}$ in $B a\left(\mathrm{Fe}_{1-x} \mathrm{Co}_{x}\right)_{2} A s_{2}$ with $x=0.074$ measured over 25 minutes. 


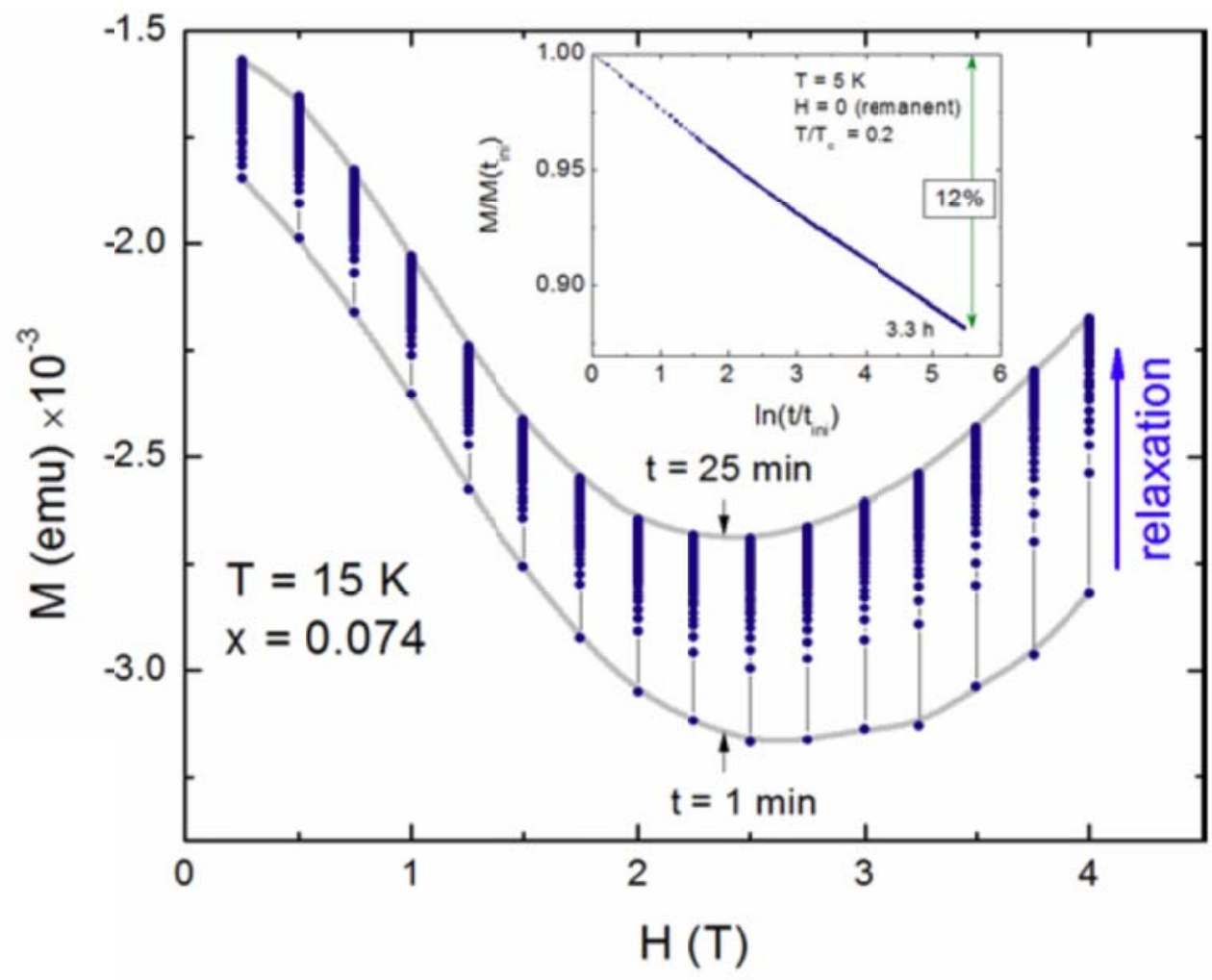

Figure 1-1: The magnetic moment relaxation as function of applied magnetic field in FeCo122 measured over 25 minutes at 15K. Inset shows the 3.3 hours relaxation decreases logarithmically with time [Prozorov et al. (2009b)].

A most interesting and commonly observed feature of unconventional superconductors is so-called second magnetization peak (also known as "fishtail") [Blatter et al. (1994)]. It has now been observed in most Fe-based superconductors when the magnetic field is aligned parallel to the crystallographic c-axis [Prozorov et al. (2008,2009); Yang et al. (2008); Kim et al.(2009); Shen et al. (2010); Pramanik et al. (2010); van der Beek et al. (2010)]. The origin of fishtail can be static, i.e., when "true" $j_{c}(H)$ is a non-monotonic function of field, $H$, or it can be dynamic 
caused by field dependent magnetic relaxation [Burlachkov, Giller, and Prozorov (1998); Mikitik and Brandt (2001)]. Experimental determination of the origin of the fishtail in each material is very important as it allows one to shed light on the nature of the flux pinning, hence defect structure "seen" by the Abrikosov vortices. In Febased superconductors, the interest is further fueled by multiple reports that defects, even non-magnetic, are pair-breaking due to presumably unconventional $s_{ \pm}$ symmetry of the order parameter [Kogan (2009); Gordon et al. (2010)]. Additionally, it seems that low-field behavior of most pnictides is governed by the so-called strong pinning, which results in a sharp peak in magnetization at $H \rightarrow 0$ [van der Beek et al. (2010)]. Therefore, to conduct a clean baseline experiment, one ideally needs Febased superconductor with reduced scattering. These materials are rare, but do exist in form of only few stoichiometric compounds, LiFeAs being one of them. Due to high sensitivity to air and moisture, there are only few reports on the vortex properties in LiFeAs crystals. The fishtail effect and relatively high $j_{B}(5 K) \approx 1 \times$ $10^{5} \frac{\mathrm{A}}{\mathrm{cm}^{2}}$ were found in [Pramanik et al. (2010)] and is shown in Figure 1-2, whereas much lower $j_{B}(5 K) \approx 1 \times 10^{3} \frac{A}{c^{2}}$ was reported in Ref.[Song et al. (2010)]. Such spread may be related to clean - limit superconductivity in this compound when even small variations of impurity concentration causes significant change in the persistent current density and magnetic relaxation. 


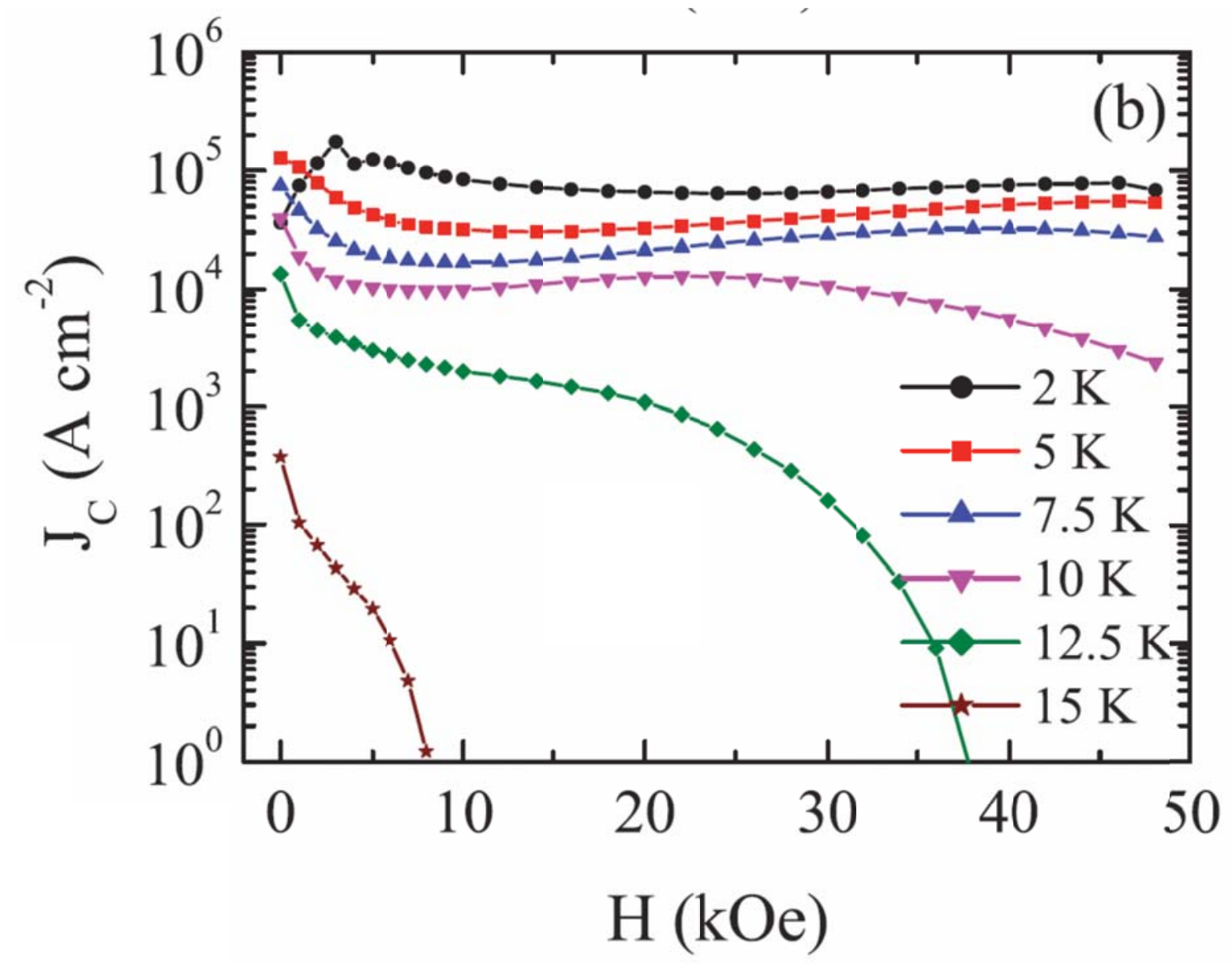

Figure 1-2: The critical current from the magnetic hysteresis loop as function of magnetic field in LiFeAs. $J_{c}(\mathrm{H})$ is nonmonotonic and exhibits the fishtail effect in the high field region [Pramanik et al. (2010)]. 


\section{Chapter 2}

\section{Labusch parameter and Campbell penetration depth}

\section{Introduction}

The macroscopic picture of a critical current in superconductors can be well explained by the Bean-critical model [Bean (1964)], while the microscopic mechanism of critical current needs to be described by the pinning force between flux lines and certain features of the microstructure. To access the information about pinning potential itself, one needs to measure the linear response when vortices are not driven out of the pinning potential well. One way to do this is to measure the socalled Campbell penetration depth, $\lambda_{C}$, which determines how far a small AC magnetic field penetrates the superconductor in the presence of vortices (induced by static external magnetic field) in the limit of $\mathrm{Hac} \rightarrow 0$, when vortex response is purely elastic and linear [Brandt (1991,1995); Koshelev and Vinokur (1991)]. This linear response regime is called the Campbell regime. The Abrikosov vortices' perturbations caused by ac magnetic field exhibit a harmonic oscillation inside a pinning potential with a pinning force directly proportional to the vortex displacement with Labusch parameter. In this chapter we define the Labusch parameter in linear response theory, explicitly express the elastic modulus in fluxoid line lattice (FLL), and elucidate Campbell penetration depth. 


\section{Labusch parameter}

When a vortex lattice is treated as perfectly rigid, the bulk pinning force due to a random array of pinning potentials approaches zero. Labusch views this as a statistical approach for calculating pinning. That is, the random pinning potentials in a very rigid array of vortices will render the directions of pinning forces in a random way which will statistically average to zero. In a real lattice, each vortex can be assumed to act individually and the global pinning force is just the direct sum of the individual forces. Labusch's original work [Labusch (1969a)] deals with the 3dimensional Green's function, delta-function, and mean field approximation which we will not elaborate here. However, the most notable review in 1-dimensional case is done by [Campbell and Evetts (2001)].

The Labusch parameter, $\alpha$, depends on lattice structure and boundary conditions and is defined as the mean of the curvature of interaction energy over vortex line elements.

$$
\alpha \equiv<\nabla \nabla U>=\left(\overline{\frac{d^{2} U_{i}}{d x^{2}}}\right)
$$

Here, $U_{i}$ is the interaction energy or pinning potential at point $i$. By statistical method, Labusch shows that the force on the flux lines per unit volume with dispersion $u_{i}$, from its equilibrium is

$$
\left(\overline{\frac{d^{2} U_{i}}{d x^{2}} u_{i}}\right)=\left(\overline{\frac{d^{2} U_{i}}{d x^{2}}}\right) \overline{u_{i}}=\alpha \overline{u_{i}}
$$


The eq. (2-2) will not be satisfied if $U_{i}$ and $u_{i}$ are statistically dependent on each other. Therefore, one must assume the individual force on each vortex is small relative to the interaction with the rest of the lattice, or in other words, the vortex is pinned randomly.

In summary, in the linear response theory, Labusch proposed that the effective pinning force, which is the summation of individual pinning interaction, can be approximated linearly by Labusch parameter $\left.\alpha \equiv \frac{d^{2} V}{d r^{2}}\right|_{r=r_{0}}$ with an effective pinning potential, $V(r)$. Clearly $\alpha$ is constant only for a parabolic $V(r)$.

\section{Elastic moduli matrix}

The elastic properties of the fluxoid line lattice in the mixed state of type II superconductors can be expressed by elastic moduli matrix which holds as the relationship between strain $\epsilon$ and stress $\sigma$. If the z-axis is arranged as the parallel axis to the flux lines, the displacement along $z$ is meaningless and thus $\epsilon_{z z}=0$. By applied symmetry conditions [Campbell and Evetts (2001); Matsushita (2007)], the elastic moduli matrix can be reduced to three independent elastic moduli described by,

$$
\left[\begin{array}{c}
\sigma_{x x} \\
\sigma_{y y} \\
\sigma_{y z} \\
\sigma_{z x} \\
\sigma_{x y}
\end{array}\right]=\left[\begin{array}{ccccc}
C_{11} & C_{12} & 0 & 0 & 0 \\
C_{12} & C_{11} & 0 & 0 & 0 \\
0 & 0 & C_{44} & 0 & 0 \\
0 & 0 & 0 & C_{44} & 0 \\
0 & 0 & 0 & 0 & C_{66}
\end{array}\right]\left[\begin{array}{c}
\varepsilon_{x x} \\
\varepsilon_{y y} \\
\varepsilon_{y z} \\
\varepsilon_{z x} \\
\varepsilon_{x y}
\end{array}\right]
$$


The tilt modulus, $C_{44}$, is applicable when the applied field are perpendicular to the surface. $C_{66}$ is the shear modulus. In which, $C_{12}=C_{11}-2 C_{66}$ where $C_{11}$ is the uniaxial compression modulus which is applicable when the flux lines are parallel to the surface of specimen. The diagram for deformation of flux line lattice for each modulus is shown in Figure 2-1.

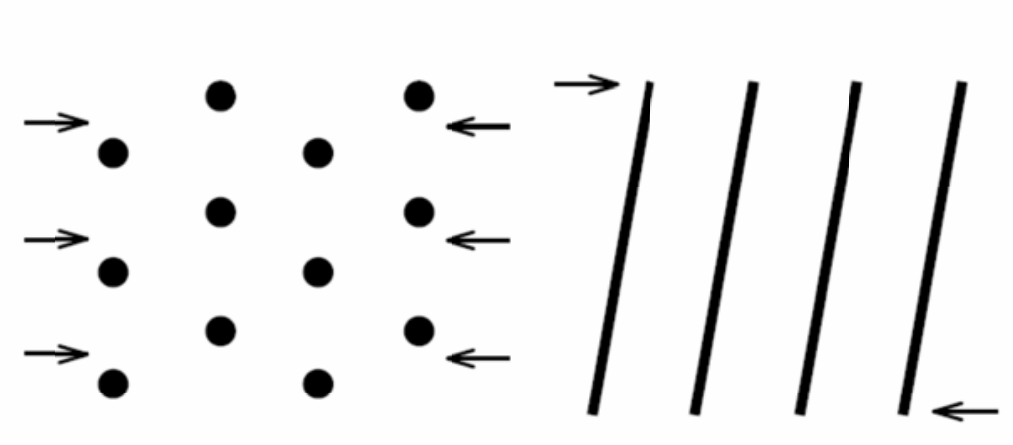

(a) (b)

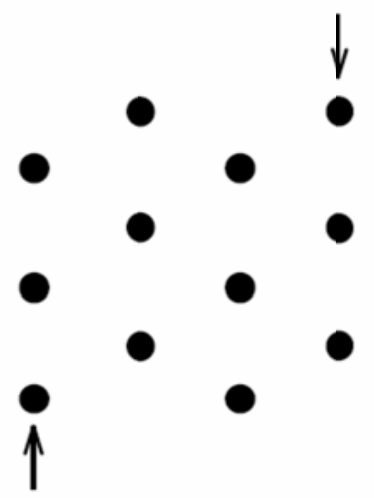

(c)

Figure 2-1: The distortion of flux line lattice corresponding to $\mathrm{C}_{11}, \mathrm{C}_{44}$, and $\mathrm{C}_{66}$, respectively [Matsushita (2007)].

The explicit expression of $C_{11}$ and $C_{44}$ was calculated in terms of magnetic field by local theory approach which considered the free energy of the fluxoid lattice [Labusch $(1698,1969)]$ or by nonlocal theory approach which considered the dispersive with respect to the wave number $k$ of flux line lattice [Brandt (1986)]. Brandt's results coincide with Labusch's as limit of $\rightarrow 0$, given as

$$
C_{11} \approx C_{44} \approx \frac{B^{2}}{4 \pi}
$$




\section{Campbell penetration depth}

In 1969, the experiment of Campbell [Campbell (1969)] first revealed the small amplitude ac magnetic field penetration depth of $\mathrm{PbBi}$ in a large dc magnetic field. In this experiment, the complete waveform signal was measured by a phase sensitive detector using a small coil wound around the sample while a small ripple ac and dc magnetic field were applied parallel to sample. The pinning force was measured at various distances from the surface. In 1971, Campbell introduced the pinning penetration depth [Campbell (1971)], which later became known as the Campbell penetration depth, in small amplitude ac response criteria where the vortex response is purely elastic and linear. The condition for Campbell regime is the effective restoring force for displacement of flux is too small to unpin a significant number of vortices. In small-amplitude ac field, when vortices are inside an averaged pinning potential, the flux lines do not drop into or jump out of the pinning potential.

In an infinite slab with uniform flux density $B$, vortices are located in equilibrium position inside the pinning potential well with no net force on the flux lines. Consider a small distortion caused by displacing the flux line with distance $u$. The pinning force from linear theory exerted on the vortex is,

$$
F=-\alpha_{L} u
$$

The magnetic flux is raised from $B$ to $B+b$ as a result of the distortion of flux line from its equilibrium. The continuity equation for flux lines [Irie and Yamafuji (1967)] is given by, 


$$
\nabla \times(B \times v)=-\frac{\partial B}{\partial t}
$$

Applying the continuity equation due to flux conservation,

$$
\frac{d u}{d x}=-\frac{b}{B}
$$

the Lorentz force from perturbation field $b$ denote,

$$
F_{L}=-\frac{B}{\mu_{0}} \cdot \frac{d b}{d x}
$$

For static equilibrium, by balancing between eq. (2-5) and eq. (2-8), we have,

$$
\frac{d^{2} b}{d x^{2}}=\frac{\mu_{0} \alpha_{l}}{B^{2}} b
$$

The solution for eq. (2-9) is

$$
\begin{gathered}
b(x)=b(0) \exp \left(-\frac{x}{\lambda_{c}}\right) \\
\lambda_{c}^{2}=\frac{B^{2}}{\mu_{0} \alpha_{L}}
\end{gathered}
$$


in cgs units

$$
\lambda_{c}^{2}=\frac{B^{2}}{4 \pi \alpha_{L}}
$$

where $\lambda_{c}$ is called Campbell penetration depth [Brandt $(1991,1995) ;$ Koshelev and Vinokur (1991); Prozorov et al.(2003)]. The result in eq. (2-10) is similar to the London penetration depth achieved from $2^{\text {nd }}$ London equation for describing the Meissner state. If the vortices are fixed due to totally rigid pinning, the superconductor will behaves as if it were in Meissner state because of the absence of vortices response from ac external field.

In general, Campbell penetration can be modified in terms of appropriate elastic modulus depending upon the geometry of the experiment [Brandt $(1991,1992)]$ as,

$$
\lambda_{C}=\left(\frac{C_{x x}}{\alpha}\right)^{1 / 2}
$$

Where $C_{x x}$ is the relevant elastic modulus corresponding to applied magnetic field, $C_{11}$ compressional modulus is for field parallel to surface, and $C_{44}$ tilt modulus is for magnetic field perpendicular to the surface. 
Therefore, in Campbell's original experiment with $B$ parallel to the surface, $\lambda_{C}=\left(\frac{C_{11}}{\alpha}\right)^{1 / 2}$. However, in our configuration, both ac and dc magnetic fields are parallel to c-axis. In other words, the magnetic fields are normal to conduction planes. Therefore the penetration occurs by tilt waves, $C_{x x}=C_{44}$

For more accuracy, by applying an exponential ansatz [Coffey and Chem $(1991,1992)]$ or Fourier analysis [Brandt $(1991)]$, the total magnetic penetration depth is

$$
\lambda_{m}^{2}=\lambda_{L}^{2}+\lambda_{C}^{2}
$$

Eq. (2-13) confirms that in the case $\alpha \rightarrow \infty$, which means the vortices are completely rigid, the magnetic penetration depth is the London penetration depth as it is in Meissner state when no vortices are present.

\section{Critical current from Campbell penetration depth}

From the Campbell penetration depth, we determine the "true" critical current density from the equation of force balance given by

$$
F_{L}+F_{P}=0
$$

where $F_{L}$ is the Lorentz force

$$
F_{L}=-\frac{B \times J}{c}
$$


and $F_{P}$ is the restoring force form the liner response

$$
F_{P}=\alpha r_{p}
$$

Here, $\alpha$ is Labusch parameter and $r_{p}$ is the effective pinning radius, usually approximated to be the coherence length $\xi$, the size of the vortex core for quenched disorder pinning at low temperatures.

With equations (2-11),(2-15), and (2-16) the critical current can be derived as,

$$
\frac{4 \pi}{c} j_{c}=\frac{r_{p} B}{\lambda_{C}^{2}}
$$

\section{Dependency of current in Campbell penetration depth}

Typical models for pinning potential in AC penetration assume that the effective potential pinning potential well is parabolic-shaped and the equilibrium flux profile is uniform depth [Campbell and Evetts (1972); Coffey and Clem (1991); Brandt (1991); van der Beek, Geshkenbein, and Vinokur (1993); Blatter et. al. (1994)]. However, if the vortex distribution is inhomogeneous, a static (Bean) current [Bean (1964)], $j_{B}$, is superimposed with the excitation ac current and the response is determined by the effective Labusch constant $\left.\alpha\left(j_{B}\right) \equiv \frac{d^{2} V}{d r^{2}}\right|_{r=r_{0}}$. Obviously $\alpha\left(j_{B}\right)$ is constant only for a parabolic $V(r)$. One possible model for current dependence is modified Labusch parameter [Prozorov $(2000,2003)]$ as

$$
\alpha=\alpha_{0}\left(1-\beta x_{0}\right)
$$


Where $\beta$ is negative if the curvature of pinning potential increases with increasing in $x_{0}$, where $x_{0}$ is the vortex distortion due to current $j$. Thus, $x_{0}=\frac{j}{j_{c}}$.

Therefore, in the presence of the Bean current, $j$, the Campbell penetration depth in eq. (2-13) can be modified to

$$
\lambda_{m}^{2}(j) \cong \lambda_{L}^{2}+\frac{\lambda_{C}^{2}(j=0)}{1-\frac{\beta j}{j_{c}}}
$$

Consider a typical experiment in which a sample is cooled in zero magnetic field and then a static magnetic field is applied. This creates a gradient of vortex density supported by the persistent Bean current density, $j_{B}$, corresponding to the first position in the pinning potential well diagram in Figure 2-2. Small-amplitude field, $H_{a c}$, causes vortex vibrations within the pinning potential well, a condition for Campbell penetration depth measurements [Brandt $(1991,1995)$; Koshelev and Vinokur (1991); Prozorov et al.(2003)]. After the sample is warmed, the vortex moves to the second position as the potential well curvature is dependent on temperature. Once the temperature exceeds the transition temperature, $T_{c}$, it is cooled again while keeping external static field constant (field-cooling) eventually reaching the third position near the equilibrium of the potential well, corresponding to $j_{B}=0$. According to eq. (2-19), we may therefore expect some hysteresis with $\lambda_{C, Z F C}>\lambda_{C, F C}$ if $V(r)$ is non-parabolic. By measuring zero field-cooled (ZFC) and field-cooled $(\mathrm{FC}) \lambda_{C}$ at different magnetic fields and temperatures we can estimate the "true" $j_{c}(H, T)$ and access information regarding shape of the pinning potential. 
For more details, the reader is referred to earlier studies of high- $T_{c}$ cuprates [Prozorov et al. (2003)].

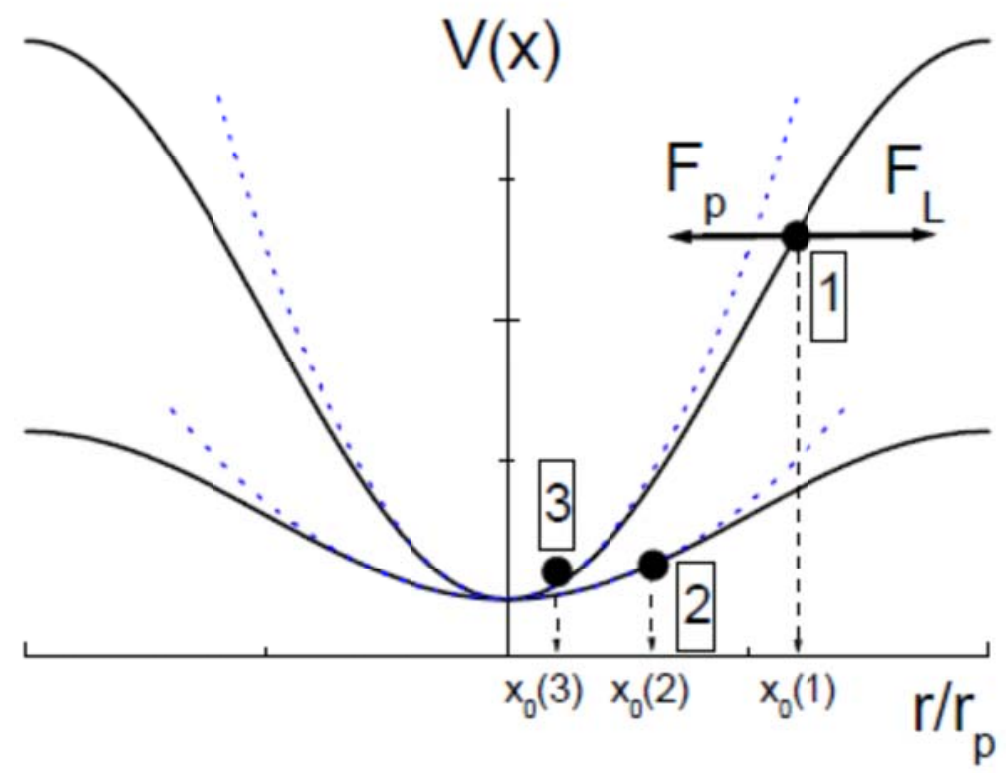

Figure 2-2: The pinning potential well diagram. Dotted lines represent the parabolic potential when Labusch parameter is constant while solid lines represent the non-parabolic potential due to gradient of vortex density. [Prozorov et al. (2003)] 


\section{Chapter 3}

\section{Tunnel-diode resonator}

In this chapter the basic principles of the tunnel-diode resonator (TDR) technique for precision measurements of magnetic penetration depth are described. The TDR experiment apparatus descripted herein is based on the setup for a ${ }^{3} \mathrm{He}$ cryostat system with DC magnetic fields up to 9 Tesla at the Superconductivity and Magnetism Low-temperature group at The Ames Laboratory, lowa State University.

\section{Introduction}

There are several ways to determine and study critical current in superconductors. Conventionally, critical current is determined from the magnetic hysteresis loop from commercial MPMS (SQUID) magnetometer device, i.e. [Pramanik et al. (2010)], or from applying Bean critical model [Bean (1964)] to dynamic visual image acquired from magneto-optic technique [Prozorov et al. (2010)], or extracted from magnetic penetration depth measured by tunnel-diode resonator (TDR) technique. The advantages of the TDR over other methods include outstanding precision and high sensitivity for detecting changes of physical properties as a function of temperature and magnetic field due to our ability to detect shifts in resonance frequency produced by changes in material properties with very 
high accuracy. Our TDR system is based on the design of [VanDegrift $(1975 b, a)]$, with a sensitivity of $0.001 \mathrm{ppm}$ in zero magnetic field measurements. Furthermore, the TDR technique is a contact-less measurement, therefore it is a very convenient technique to use for measuring a wide range of properties such as magnetic properties and transitions in ferromagnetic and antiferromagnetic materials [Vannette et al. (2008b)], absolute London penetration depth in novel FeAs based superconductors [Gordon et al. (2010b)], and quantum oscillation in metals [Prozorov et al. (2006)].

\section{TDR circuit component}

The basis of the TDR method is a self-resonant LC circuit. The simple circuit diagram is shown in Figure 3-1: The circuit part of tunnel diode resonator. The values for the circuit components are so chosen as to achieve the optimal condition for stability at operating temperature. Inductor coil and capacitors are used to establish the resonance frequency. The values of components in our home-built TDR circuit operating in ${ }^{3} \mathrm{He}$ cryostat are shown in Table 3-1.

The resonance of the LC circuit is maintained by power from the tunnel diode which compensates for energy dissipation. However, the tunnel diode provides just enough energy for self-resonance of the LC circuit. To achieve this, the supplied bias voltage is precisely set in the region of negative differential resistance. The IV characteristic curve is plotted in Figure 3-2: The characteristic IV curve for Areroflex/Metelics BD3 tunnel diode. The operating bias voltage is kept in the region with negative differential resistant so the diode only provides marginal energy to 
keep a self-resonant LC circuit [Gordon (2011)].. The operating bias voltages is kept in the region with negative differential resistant so that diode only provide marginal energy to keep self-resonant LC circuit [Gordon (2011)].

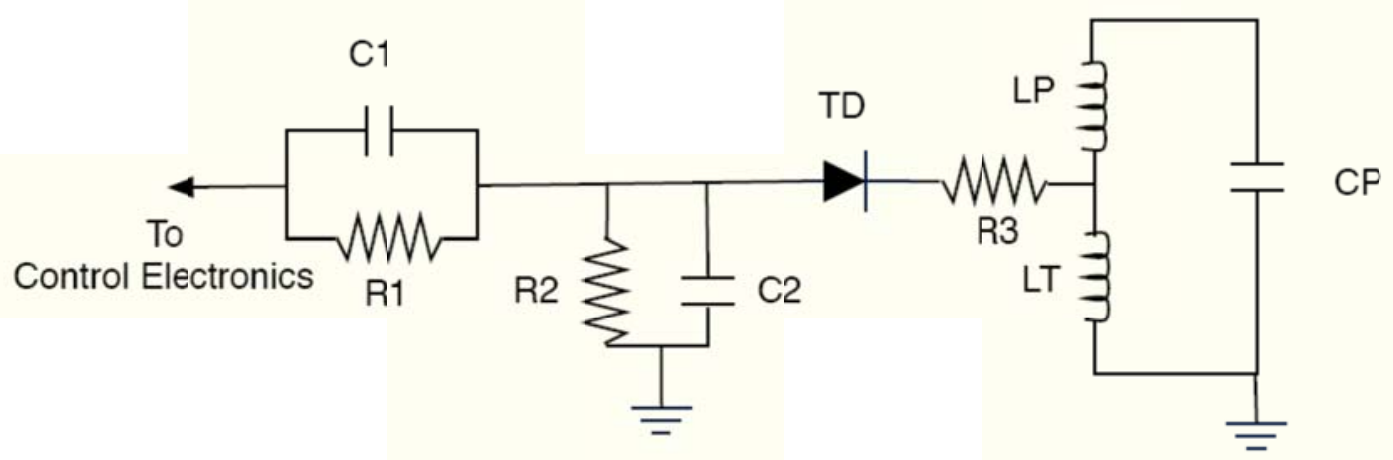

Figure 3-1: The circuit part of tunnel diode resonator [ Vannette (2008) ].

Table 3-1: Values of the TDR circuit component in diagram

\begin{tabular}{|c|c|}
\hline Label & Value \\
\hline R1 & $1500 \Omega$ \\
\hline R2 & $300 \Omega$ \\
\hline R3 & $300 \Omega$ \\
\hline C1 & $22 \mathrm{pF}$ \\
\hline C2 & $0.012 \mu F$ \\
\hline CP & $120 \mathrm{pF}$ \\
\hline
\end{tabular}




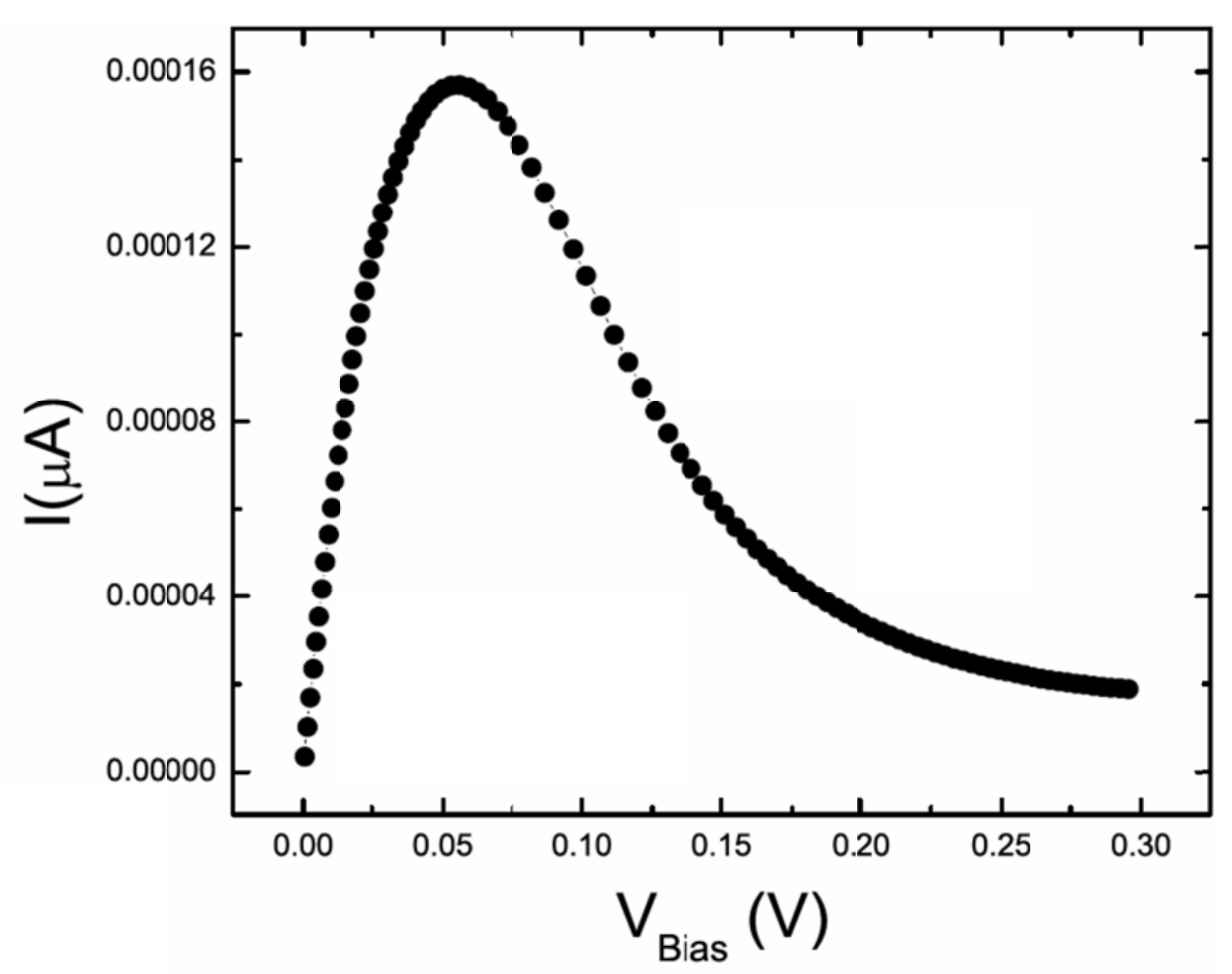

Figure 3-2: The characteristic IV curve for Areroflex/Metelics BD3 tunnel diode. The operating bias voltage is kept in the region with negative differential resistant so the diode only provides marginal energy to keep a self-resonant LC circuit [Gordon (2011)].

Both the primary coil (LP), and the trap coil (LT) are $2 \mathrm{~mm}$ diameter solenoid hand-wound using AWG40 insulated copper wire. The number of turns are 60 and 20 respectively. An even separation between neighboring turns is obtained by winding two wires around a drill bit then unwinding one. Finally, the coils rigidly are rigidly set using Stycast 1266 epoxy. The ratio between these two coils corresponds to VanDegrift's design in order to suppress contributions from higher 
harmonic frequencies within the resonance frequency spectrum. Samples are placed on top of a sapphire rod and inserted into the center of the primary coil. The LC circuit produces an rf excitation field (at $f \approx 14 \mathrm{MHz}$ ) of $H_{a c} \sim 20$ mOe at the center of the primary coil. An external DC magnetic field $(0-9 \mathrm{~T})$ is applied parallel to the AC field, both parallel to the $c$ - axis, i.e. $H_{a c}\|H\| c$-axis. The diagram for the experimental configurations of our TDR technique is presented is Figure 3-3.

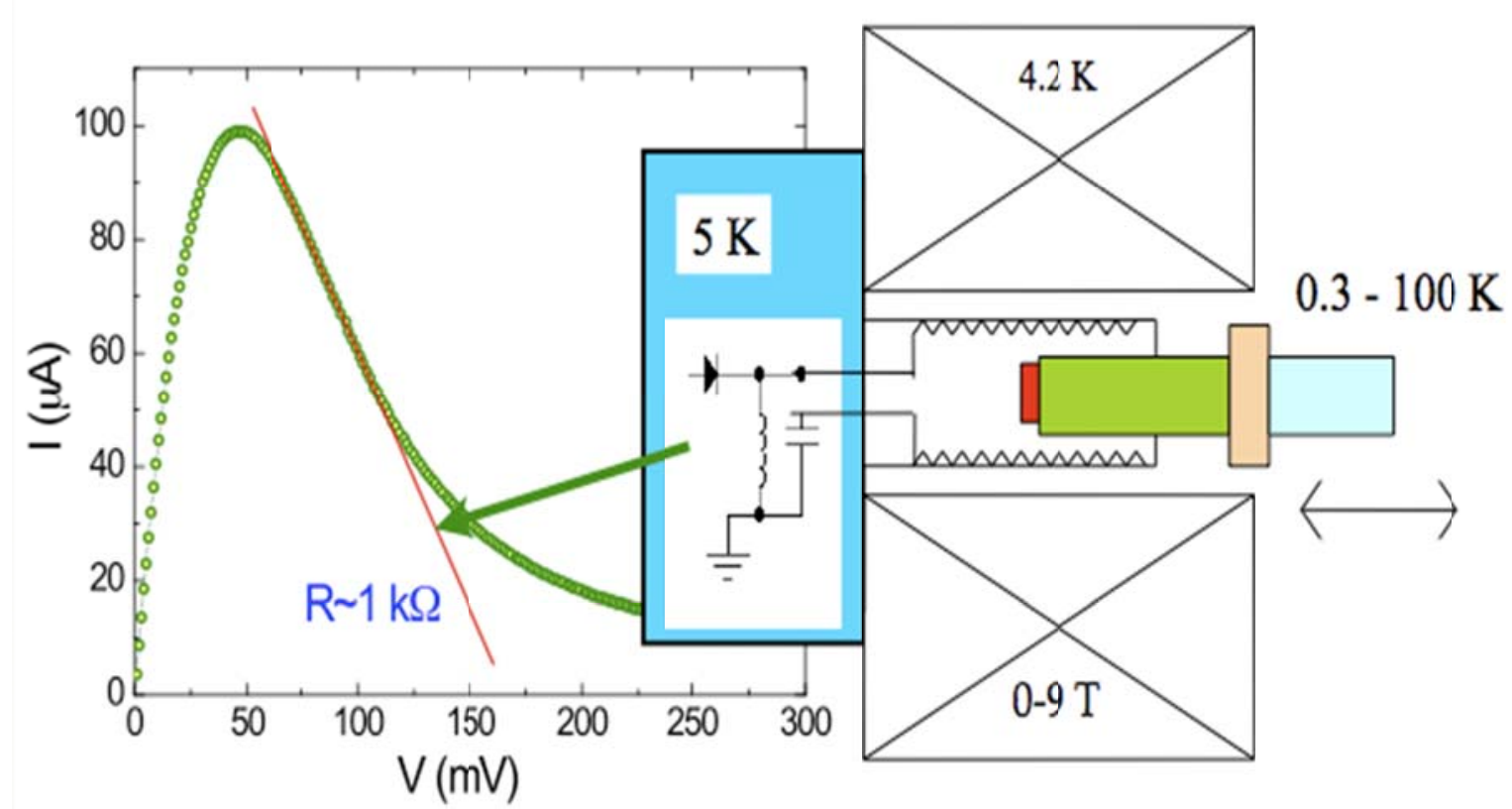

Figure 3-3: Schematic of the TDR technique apparatus [Prozorov and Giannetta (2006)].

\section{Magnetic penetration depth measurement}

The magnetic penetration depth is measured by detecting a shift of the resonance frequency which results from a change of sample properties inside the 
primary coil. In this part, we will elaborate how this shift relates to the properties of the sample and the calibration for the magnetic penetration depth. First, the general expression for resonance frequency for a typical LC circuit is in eq. (3-1)

$$
f_{0}=\frac{1}{2 \pi \sqrt{L C}}
$$

where $L$ is the inductance from the primary coil and $C$ is the efficientive capacitance of the TDR circuit. Let us consider a small change in inductance, $\Delta L$, from $L$ to $L^{\prime} \equiv L+\Delta L$ as a response to the changes of sample properties. The new resonance frequency will be

$$
f_{0}+\Delta f=\frac{1}{2 \pi \sqrt{(L+\Delta L) C}}
$$

If $\Delta L / L \ll 1$, by using the partial differential property $d f[L, C]=\partial_{L} f d L+\partial_{C} f d C$ [Arfken and Weber (2005)], the small increment in frequency can be cast as

$$
\frac{\Delta f}{f}=-\frac{\Delta \mathrm{L}}{2 L}
$$

with the definition of inductance,

$$
L=\frac{d \phi}{d I}
$$

Here, the magnetic flux is $\phi=H V_{c}$, where $\mathrm{H}$ is the applied field produced by the primary coil and $V_{c}$ is the volume of the coil. 
If the sample is inserted, the inductance becomes

$$
L^{\prime}=\frac{d \phi^{\prime}}{d I}
$$

The magnetic flux can be expressed by

$$
\phi^{\prime}=H\left(V_{c}-V_{s}\right)+B V_{s}
$$

where $V_{s}$ is the sample volume and $B$ is the magnetic field inside of the sample.

Recall, that

$$
B=H+4 \pi M \text { (in cgs units), }
$$

$\phi^{\prime}$ can be rewritten as

$$
\phi^{\prime}=H V_{c}+4 \pi V_{s} M
$$

Applying the definition in eq. (3-5) to eq. (3-8)

$$
\frac{\Delta L}{L}=\frac{4 \pi V_{s}}{V_{C}} \chi
$$

where $\chi$ is the ac magnetic susceptibility of the sample.

Combining eq. (3-3) and (3-9), one finally reaches an expression for a shift of the resonant frequency (in cgs units):

$$
\frac{\Delta f}{f}=-\frac{V_{s}}{2 V_{c}} 4 \pi \chi
$$


With the analytical geometry studied and the effective dimension, [Prozorov et al. (2000); Prozorov and Giannetta (2006)], an approximation of susceptibility $\chi$ accounting for demagnetization is given by

$$
-4 \pi \chi=\frac{1}{1-N}\left[1-\left(\frac{\lambda}{R}\right) \tanh \left(\frac{R}{\lambda}\right)\right]
$$

where $N$ is the demagnetization factor. $\lambda$ is the magnetic penetration depth. The characteristic sample size, $R$ is defined as

$$
R_{3 D}=\frac{w}{2\left(1+\left[1+\left(\frac{2 d}{w}\right)^{2}\right] \arctan \left(\frac{w}{2 d}\right)-\frac{2 d}{w}\right)}
$$

where $w=\frac{a b}{a+b}$. The variables used in eq. (3-12) are marked in the geometry of a hypothetical sample in Figure 3-4.

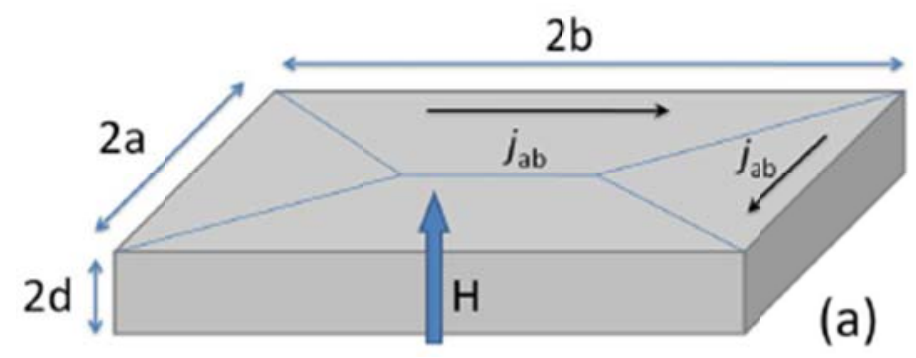

Figure 3-4: Geometry relevant to equation (3-12). The applied DC magnetic field is arranged parallel to the c-axis of specimen [Prozorov et al. (2008)]. 
Therefore, by combining eq. (3-3), (3-9), and (3-11) the shift of the resonant frequency (in cgs units) is given by

$$
\begin{gathered}
\Delta f(T)=-G\left[1-\left(\frac{\lambda}{R}\right) \tanh \left(\frac{R}{\lambda}\right)\right] \\
G \equiv \frac{f_{0} V_{s}}{2 V_{c}(1-N)}
\end{gathered}
$$

where $G$ is defined as the calibration constant which is determined from the full frequency change by physically pulling the sample out of the coil.

For actual sample, in the case $T<T_{c}$, where $T_{c}$ is the transition temperature and in the limit of $R \gg \lambda$,

$$
\tanh \left(\frac{R}{\lambda}\right) \rightarrow 1
$$

So eq. (3-13) becomes

$$
\Delta f(T)=-G\left[1-\left(\frac{\lambda}{R}\right)\right]
$$

Therefore, the change in $\lambda(\Delta \lambda)$ with respect to its value at low temperature, can be obtained by solving eq.(3-14) in the limit of eq. (3-15), as

$$
\Delta \lambda=\delta f\left(\frac{R}{G}\right)
$$

where $\Delta \lambda \equiv \lambda(T)-\lambda\left(T_{\min }\right)$ and $\delta f \equiv \Delta f(T)-\Delta f\left(T_{\min }\right)$ 
We can determine the London penetration depth $\lambda_{L}(T)$ from measurements at $H=0$. In particular, if the absolute value of London penetration depth $\left(\lambda_{L}(T=0) \equiv\right.$ $\lambda(T=0, H=0))$ is known then $\lambda\left(T_{\min }\right)$ is shifted to value $\lambda_{L}(T=0)$, and $\Delta \lambda$ can be standardized as $\lambda(T)$. For the magnetic penetration depth $\lambda_{m}(T)$ which is measured in applied DC magnetic field, the calibrated $\lambda_{m}(T)$ can be achieved by shifting $\Delta \lambda$ in the normal state to be the same value as $\lambda(T)$ in $H=0 \mathrm{~T}$. At the normal state, the penetration depth converts to the electromagnetic skin depth limit

$$
\delta=\sqrt{\frac{2 \rho}{\mu \omega}}
$$

Further, the Campbell penetration depth can also be extracted since the measured penetration depth in applied DC magnetic field by TDR consists of the London penetration depth and Campbell penetration depth by $\lambda_{m}^{2}=\lambda_{L}^{2}+\lambda_{C}^{2}$ [Brandt (1995)].

\section{Background noise and measurement offset}

In general, TDR technique is geared to measuring the absolute London penetration depth in zero applied magnetic field. However, for the measurement of magnetic penetration depth, the 0-9T DC magnetic field is applied. Therefore, it is worthwhile elaborating on the noise in TDR since both the circuit and the sample are in the magnetic field.

First, the drift of the resonance frequency is about 2-3 Hz over $45-50$ minutes. This drift is neither a function of magnetic field nor a function of sample temperature 
as shown in Figure 3-5. Next, the field dependence for an empty run is plotted in Figure 3-6. The resonant frequency of TDR circuit is field-dependent, i.e. if the applied magnetic field changes, the resonance frequency also changes. However, the resonant frequency is not related to the sample temperature because of the highly effective thermal isolation between the sample holder and TDR circuit. This shift in resonance frequency is quite reproducible across different samples, thus we can subtract it as background measurement offset. 


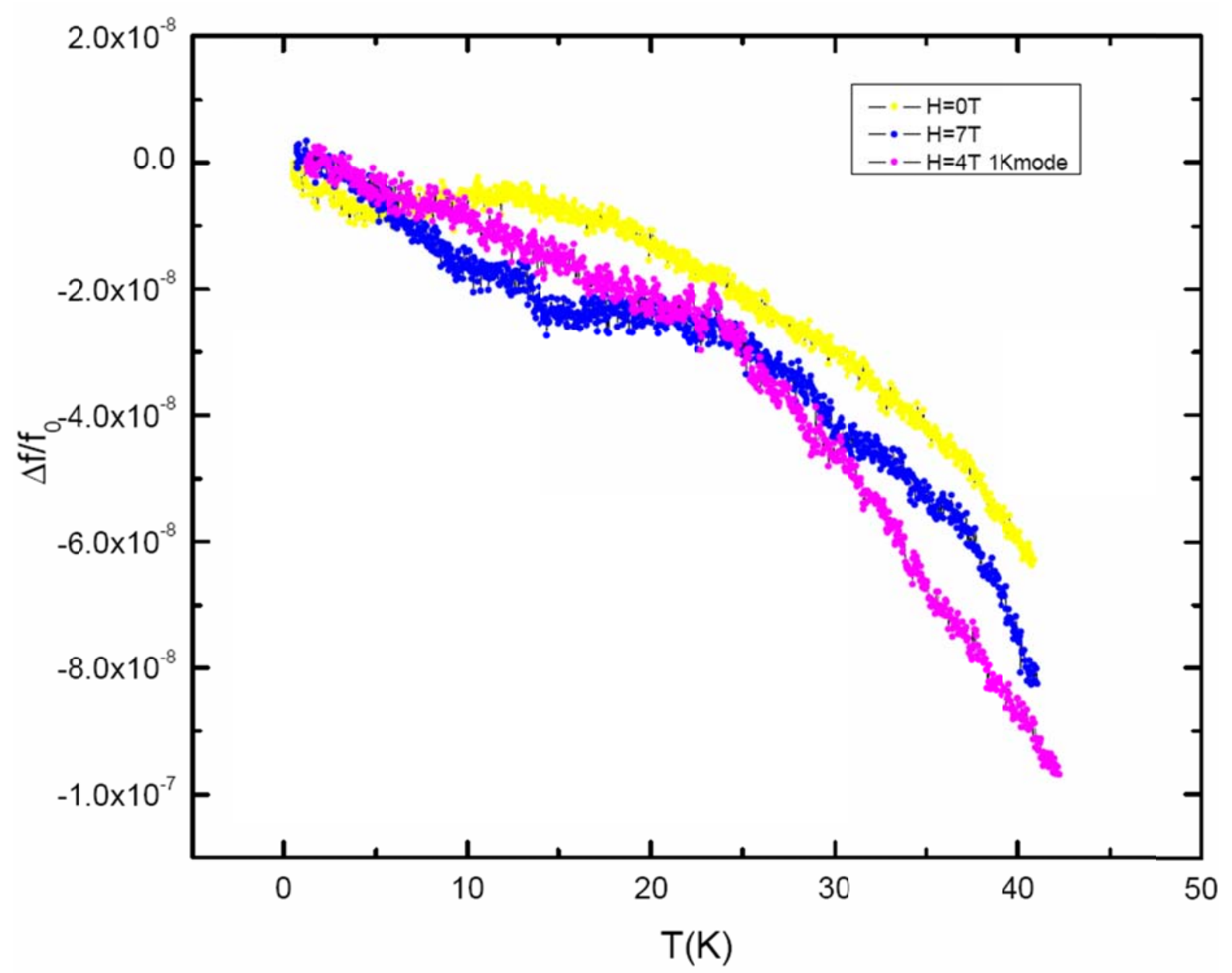

Figure 3-5: The frequency drift as a function of temperature at $\mathrm{H}=0,4,7$ Tesla. The period for each temperature sweep experiment was about 45 minutes. The drift is less than $2 \mathrm{~Hz}$. 


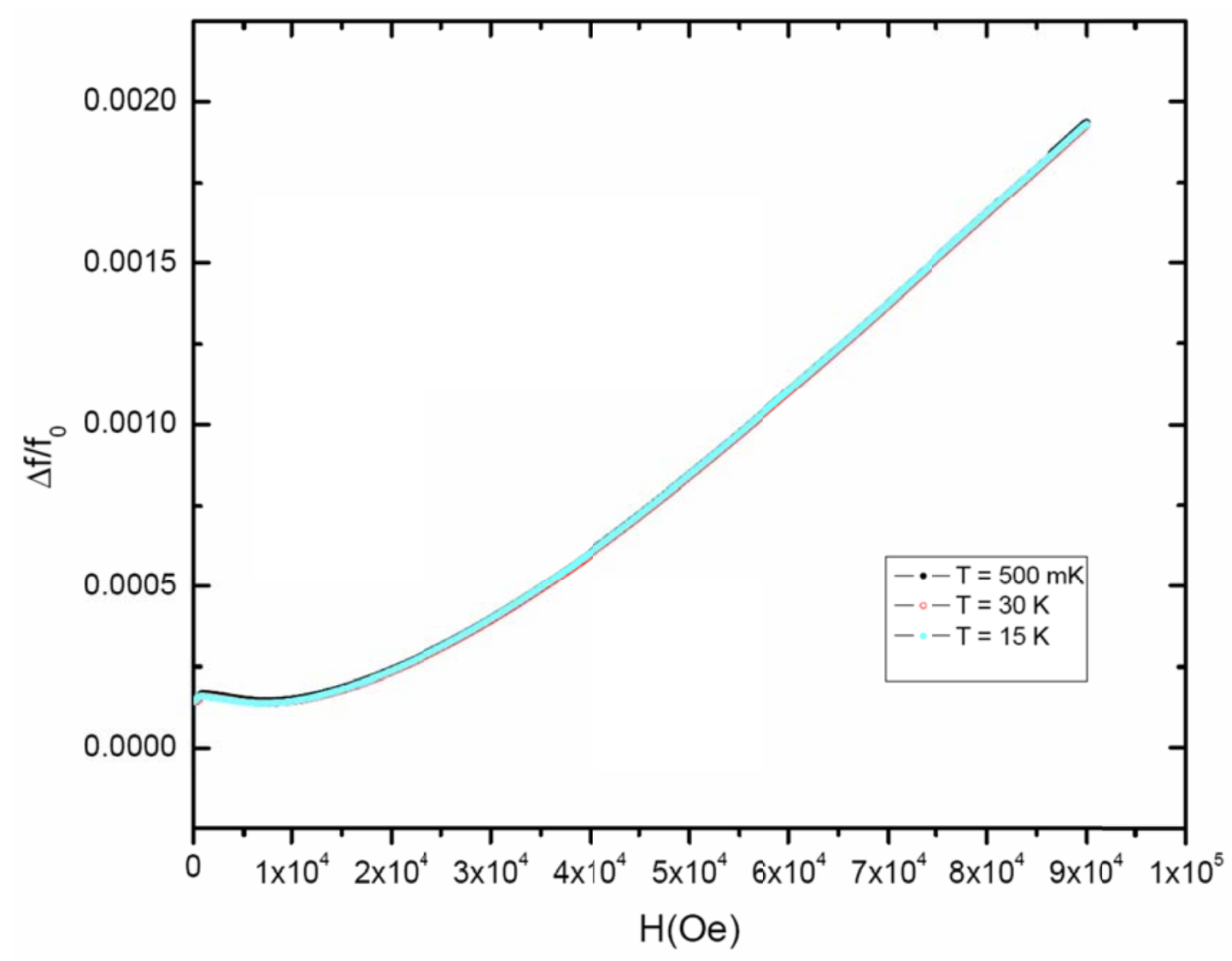

Figure 3-6: The shift in frequency as a function of applied DC magnetic field at $\mathrm{T}=0.5,15,30 \mathrm{~K}$. 


\title{
Chapter 4
}

\section{Campbell penetration depth in LiFeAs superconductor}

\begin{abstract}
The discussion in this chapter is based on the work [Prommapan et al. (2011)]

In this chapter we report measurements of Campbell penetration depth in single crystals of LiFeAs. We show that the fishtail has dynamic origin and the fielddependent magnetic relaxation is due to transformation of the pinning potential with field. Namely, Labusch constant (and "true" critical current, $j_{c}(H)$ ) is a monotonic function of field when Bean current (macroscopic vortex density gradient) is present, but it becomes a nonmonotonic function of field at a homogeneous distribution of vortices. The values of $j_{c}(2 K) \approx 1.22 \times 10^{6} \frac{\mathrm{A}}{\mathrm{cm}^{2}}$ provide upper estimate of the current carrying capability of this material and show the significance of magnetic relaxation. We also find evidence for the strong pinning regime at the low fields. With the increase of the magnetic field vortex pinning and creep change to a collective regime and, finally, cross over to another vortex state, perhaps dominated by plastic deformations. Despite being quite different from high- $T_{c}$ cuprates in terms of pairing and gap structure, it seems that vortex behavior of Fe-based superconductors is remarkably similar to high- $T_{c}$ materials.
\end{abstract}




\section{Sample and method}

Single crystals of LiFeAs were grown out of Sn flux as described in detail elsewhere [Lee et al. (2010)] and were transported for measurements in sealed ampoules. Immediately after opening, $(0.5-1) \times(0.5-1) \times(0.1-0.3) \mathrm{mm}^{3}$ samples were placed into the cryostat for the measurements. Additionally, samples were extensively characterized by transport and magnetization measurements [Lee et al. (2010)]. Zero-field transition temperature of our samples was about, $T_{c} \approx 18$ $\mathrm{K}$. The magnetic penetration depths were measured by TDR technique as discussed in chapter 3.

\section{Results and discussion}

Figure 4-1 shows magnetic penetration depth measured upon warming, after sample was cooled in zero field and target field was applied at low temperature (ZFC-W) compared to the measurements upon cooling when target field was fixed above $T_{c}$ and kept constant (FC-C). A step at low temperatures on a $H=0$ curve is due to residual Sn flux. It was quenched by applying a moderate $H=250$ Oe field, which does not affect our analysis of the much higher fields. Inset in Figure 4-1 shows an example of the magnetic hysteresis measured at $H=7 \mathrm{~T}$ (notice that once ZFC-W process was complete, subsequent warming-cooling measurements (FC-C and FC-W) resulted in the same curve indicating homogeneous vortex distribution). The hysteresis between ZFC-W and FC-C-W is much smaller than, for example, observed in BSCCO crystals [Prozorov et al. (2003)], which is most likely due to much more 3D electronic nature of LiFeAs. From the measured penetration 
depth in zero field, $\lambda_{L}(T)$, and the one measured in applied magnetic field, $\lambda_{m}(T, H)$, we determine the Campbell penetration depth via, $\lambda_{C}=\sqrt{\lambda_{m}^{2}-\lambda_{L}^{2}}$ as shown in Figure 4-2.

From the Campbell penetration depth we determine the "true" critical current density as, $\frac{4 \pi}{c} j_{c}=\frac{r_{p} \phi_{0}}{\lambda_{C}^{2}}$ were we assumed the radius of the pinning potential be a coherence length, $r_{p} \simeq \xi \simeq 4.4 \mathrm{~nm}$. This estimate for $\xi$ comes from the measurements of the upper critical field $H_{c 2}(0) \simeq 17 \mathrm{~T}$ [Cho et al. (2011)], but $\xi \simeq 7 \mathrm{~nm}$ has been reported from neutron scattering form factor [Inosov et al. (2010)]. Figure 3 shows $j_{c}$ as a function of temperature at different magnetic fields determined after ZFC-W process (top frame) and FCC process (bottom frame). In both cases, the curves are monotonic in temperature and show substantial temperature dependence similar to high $-T_{c}$ cuprates, re-enforcing the earlier statement that vortex properties of Fe-based superconductors are remarkably similar to the cuprates, despite the difference in dimensionality of the electronic structure [Tanatar et al. (2009)].To understand the functional dependence, we plot determined $j_{c}(T)$ on a semi-logarithmic plot as shown in the insets in Figure 4-3. At relatively low fields, the behavior is very similar to the earlier reports of strong pinning [van der Beek et al. (2010)] and can be well approximated by the exponential temperature dependence, $j_{c}(1 T) \simeq 2.1 \exp \left(\frac{-T}{3.1}\right) \frac{\mathrm{MA}}{\mathrm{cm}^{2}}$ for FC-C process and $j_{c}(1 T) \simeq$ $2.3 \exp \left(\frac{-T}{3.2}\right) \frac{\mathrm{MA}}{\mathrm{cm}^{2}}$ for ZFC-W measurements. This very similar behavior imply that strong pins result in a more-or less parabolic $V(r)$ and are practically independent of the bias Bean current, $j_{B}$. However, at the higher fields, the critical current becomes 
less temperature dependent, probably due to saturation of strong pins and a crossover first to the collective pinning regime and eventually to the disordered lattice dominated by plastic deformations. Finally, Error! Reference source not found. shows "true" critical current density, $j_{c}$, determined form ZFC Campbell penetration depth (top frame) and from the FC Campbell penetration depth (bottom frame) as a function of magnetic field at different temperatures. While ZFC curves are monotonic, a clear fishtail signature is observed in the equilibrium FCC-W measurements at higher temperatures. The inset in Error! Reference source not found. emphasizes this result.

\section{Conclusions}

Our results can be interpreted in the following way. Maximum critical current values, $j_{c}(2 \mathrm{~K}) \approx 1.22 \times 10^{6} \frac{\mathrm{A}}{\mathrm{cm}^{2}}$, show that conventional measurements underestimate critical currents, probably due to significant magnetic relaxation. However, the most striking result is that $j_{c}$, obtained in a non-equilibrium ZFC process, is monotonic with magnetic field at all temperatures, whereas equilibrium $j_{c}$, obtained in the FC process where the flux profiles inside the sample is uniform, shows a clear signature of the fishtail (second peak) magnetization. (Note that FC $j_{c}$ is only a convenient parameter characterizing the pinning potential and does not represent the current density that can be measured.) Since conventional (relaxed) DC measurements show fishtail [Pramanik et al. (2010)], we conclude that fishtail effect is of dynamic origin, which means that magnetic relaxation is faster at the lower fields. Moreover, during relaxation the effective vortex pinning potential 
transforms, probably indicating collective effects and ultimately a crossover to the disordered vortex lattice. It is possible that fishtail has similar origin in high temperature cuprates and a very interesting question is how to reconcile a very different (almost isotropic) electronic properties of Fe-based superconductors and quite similar to highly anisotropic cuprates vortex behavior.

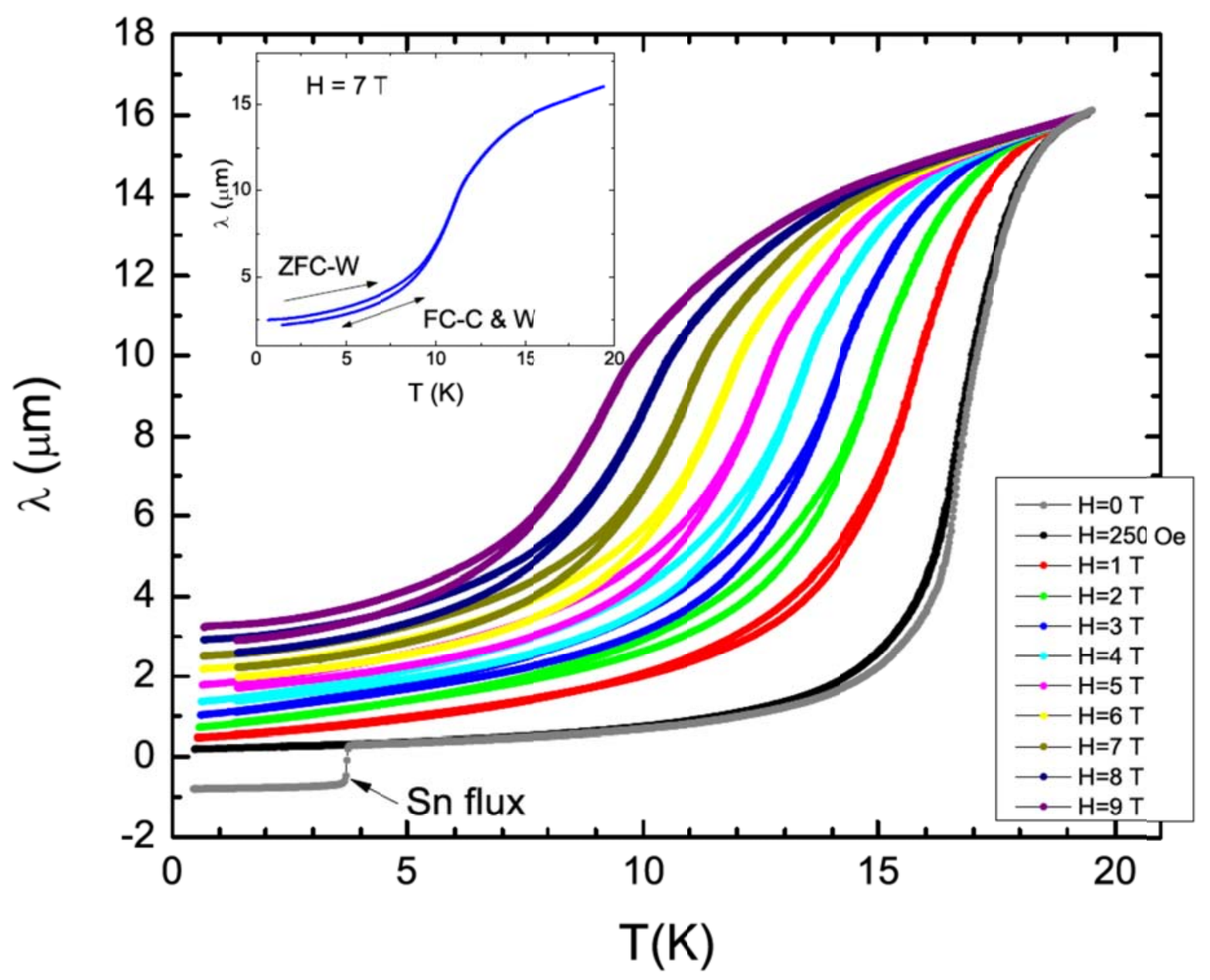

Figure 4-1: Magnetic penetration depth measured in a ZFC-FC process at different fields. $H=0$ curve shows a step due to leftovers of Sn flux. It was quenched by applying a $H=250$ Oe field. Inset shows an example of the hysteresis of $\lambda_{m}(T)$ at $H=7 \mathrm{~T}$. 


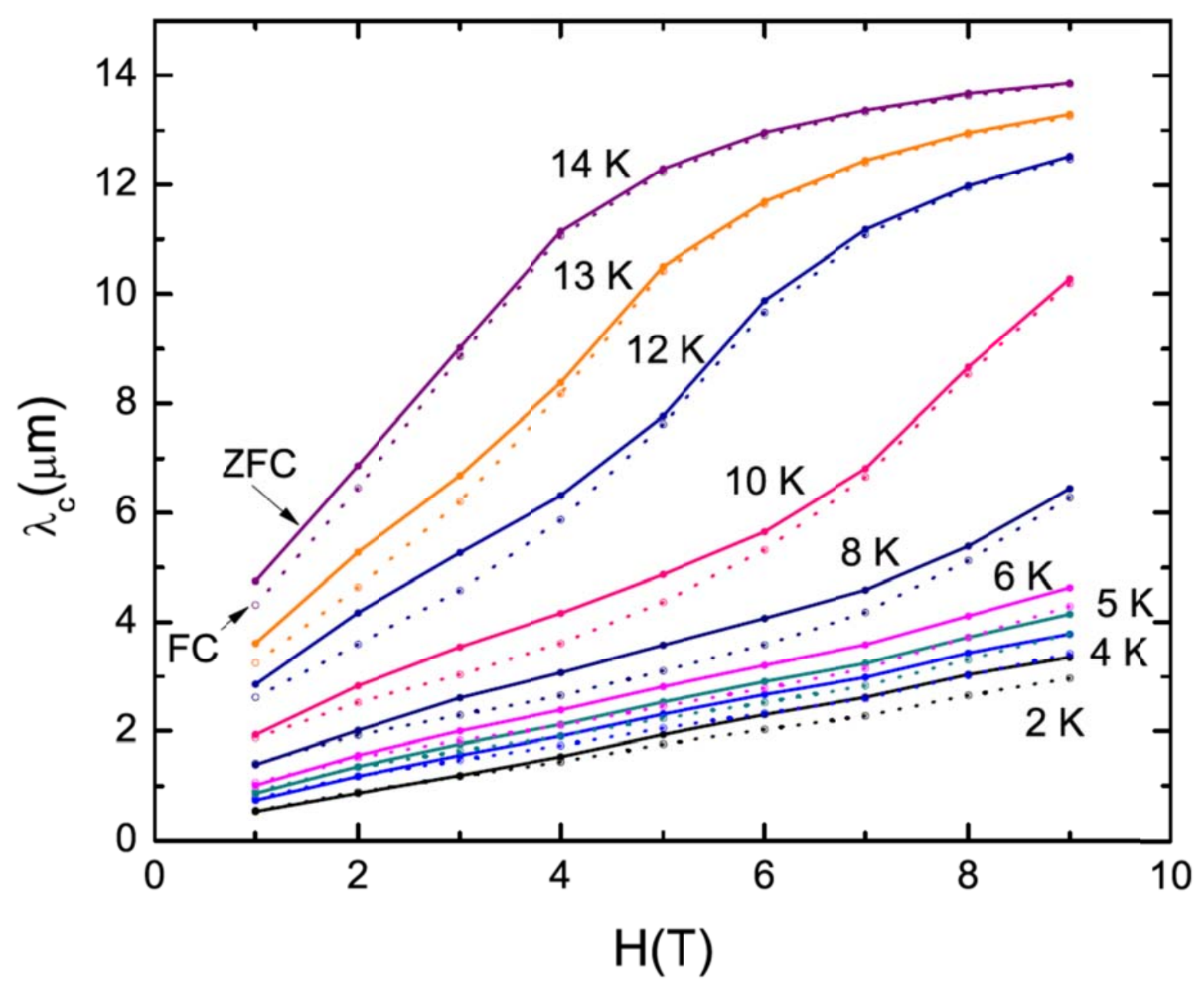

Figure 4-2: Campbell penetration depth as function of magnetic field at different temperatures extracted from the data of Fig. 1. Solid lines - ZFC and dashed lines are FC data. 


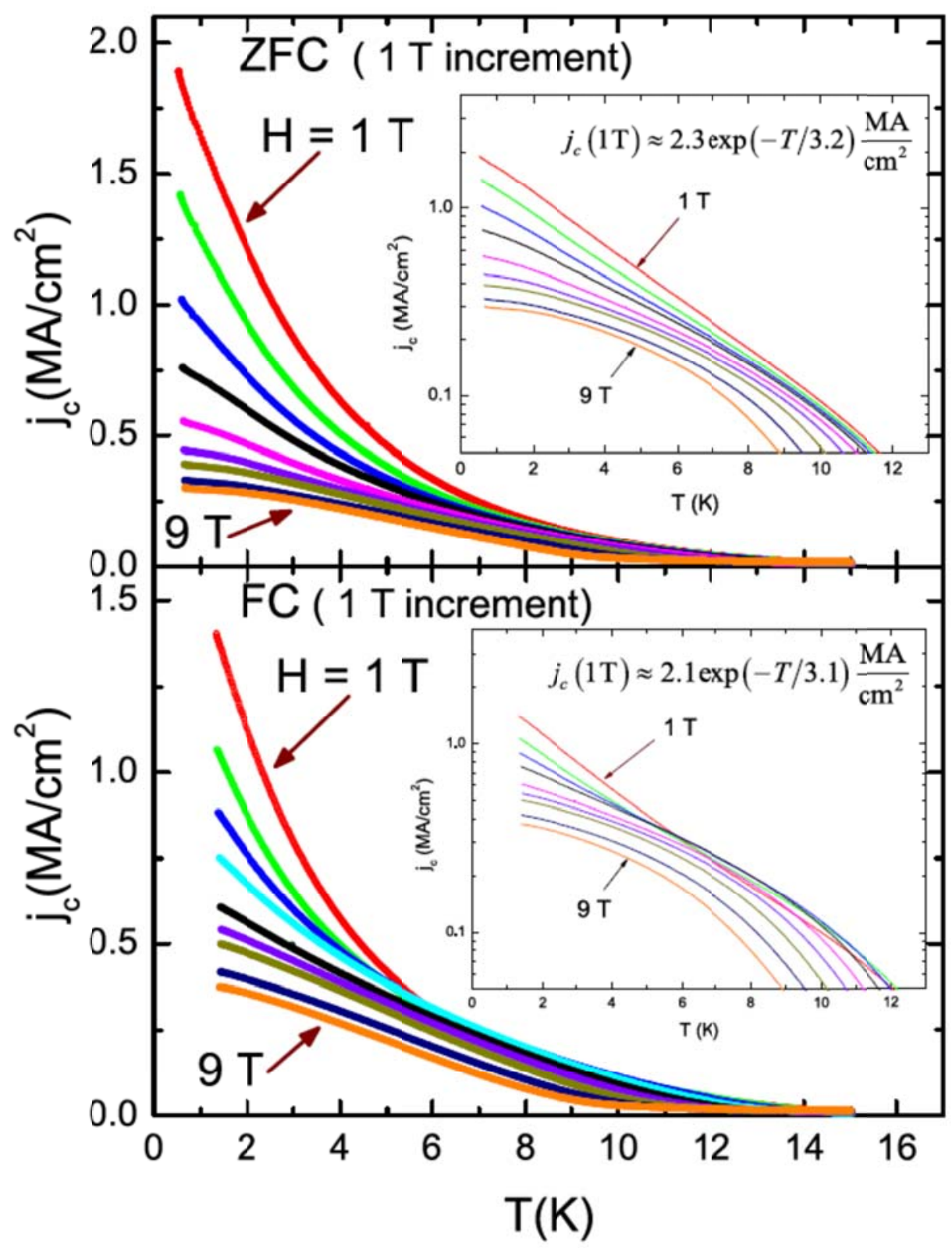

Figure 4-3: "true" critical current density, $\boldsymbol{j}_{\boldsymbol{c}}$, determined from the ZFC (top frame) and FC (bottom frame) experiments at indicated values of the applied external magnetic fields. Insets show semi-log plots indicating exponential dependence of $\boldsymbol{j}_{\boldsymbol{c}}$ at lower fields and a crossover to a different pinning regime at the higher fields. 


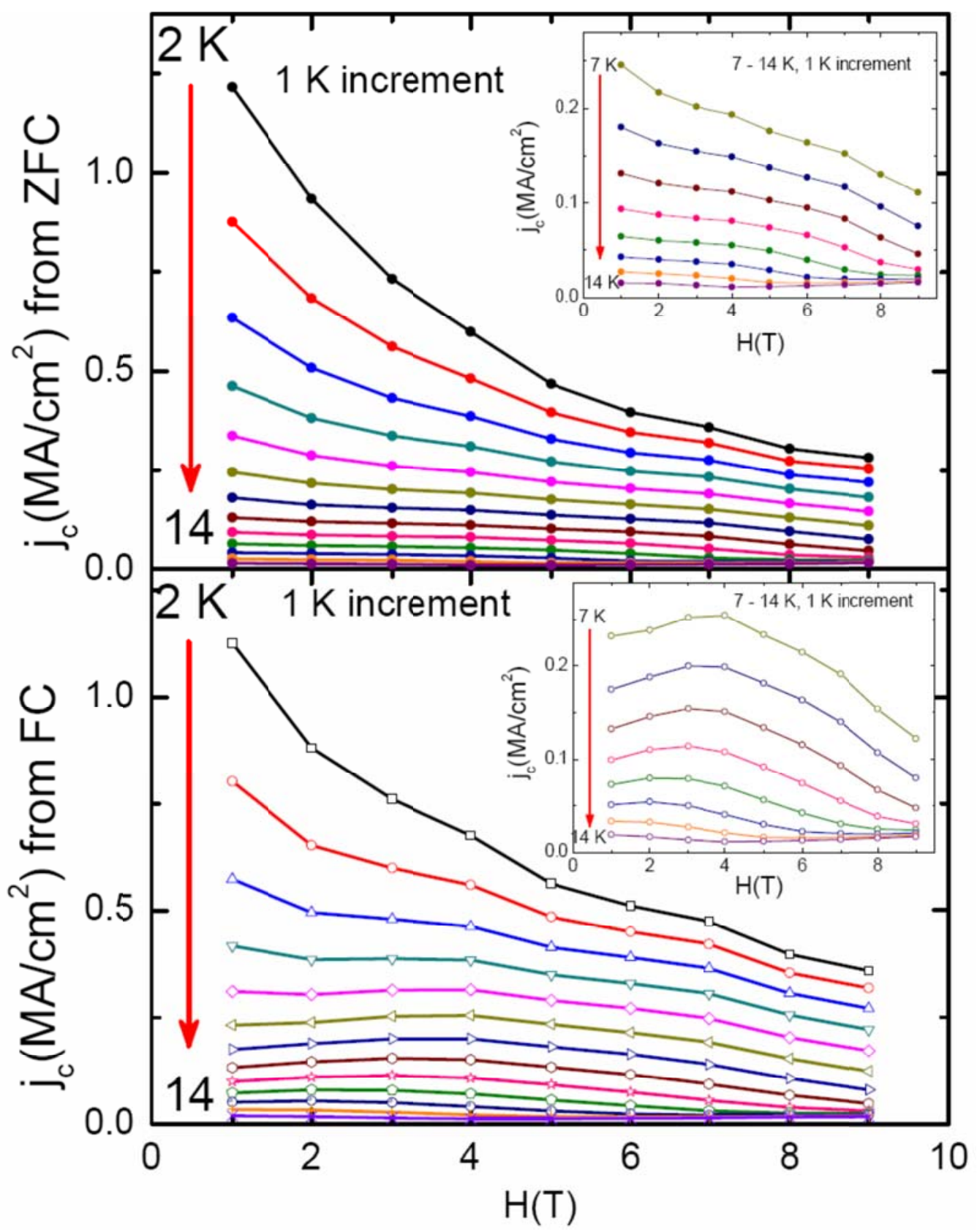

Figure 4-4: Critical current density determined form ZFC Campbell penetration depth (top frame) and from the FC Campbell penetration depth (bottom frame) showing the absence of the fishtail magnetization in the former and its presence in the latter. 


\section{Chapter 5}

\section{Campbell penetration depth in other Fe-based superconductor}

In the last chapter, we extensively investigated the field and temperature dependence of penetration depth for a small ac field in the 111 pnictide superconductor LiFeAs. The Labusch parameter and consequently the temperatureand field-dependent "true" critical current densities in ZFC and FC measurements were derived from the Campbell penetration depth. The origin of the fish tail effect observed in LiFeAs was discussed. In this chapter, we apply a similar procedure and extend our report of magnetic penetration depth to other families of Fe-base superconductor

$\mathrm{Ba}\left(\mathrm{Fe}_{0.954} \mathrm{Ni}_{0.046}\right)_{2} \mathrm{As}_{2}$

First, we measured magnetic penetration depth in two samples of singlecrystalline optimally electron-doped $\mathrm{Ba}\left(\mathrm{Fe}_{0.954} \mathrm{Ni}_{0.046}\right)_{2} \mathrm{As}_{2}$ (FeNi122) where the first sample, C0 is unirradiated sample and the second sample, C3 is irradiated sample with $1.4 \mathrm{GeV}^{208} \mathrm{~Pb}^{56+}$ ions with the irradiation dose of $B_{\phi}=2 \mathrm{~T}$. Single crystals of FeNi122 were grown out of FeAs flux using high temperature solution growth technique whose details and physical characterization can be found in [Ni et al. (2008); Canfield et al. (2009); Bud'ko, Ni, and Canfield (2009)]. C0 and C3 share the 
same dimensions of $0.7 \times 0.93 \times 0.02 \mathrm{~mm}^{3}$. The critical current densities of both samples were previously studied using Magneto-optic (MO) imaging and estimated from the Bean model. [Prozorov et al. (2010)]. The results from MO imaging of both $\mathrm{C} 0$ and $\mathrm{C} 3$ are shown in Figure 5-1. The magnetic penetration depths were measured by TDR technique as discussed in chapter 3 .

\section{Unirradiated $\mathrm{Ba}\left(\mathrm{Fe}_{0.954} \mathrm{Ni}_{0.046}\right)_{2} \mathrm{As}_{2}$}

First, we begin with the unirradiated FeNi-122 (C0 sample) as the reference. Figure 5-2 shows magnetic penetration depth measured upon warming, after sample had been cooled in zero field and target field had been applied at low temperature (ZFC process). Consequently, after the sample had been warmed above $T_{c}$, measurements upon cooling continued in the same applied field (FC process). The zero-field transition temperature of C0 sample is about $T_{c}=18 \mathrm{~K}$. There is no significant hysteresis between ZFC and FC processes. This result implies that the pinning potential $V(r)$ is likely to be of parabolic shape. Also, Campbell penetration depth $\lambda_{c}$ does not significantly depend upon Bean current, $j_{B}$. As a result, we will content ourselves with investigating only ZFC process as it is not vastly different from FC. Figure 5-3 shows $\lambda_{c}$ as a function of magnetic field at various temperatures. From the Campbell penetration depth we determine the critical current density by identifying the radius of the pinning potential to be the coherence length $\xi \approx 2.44 \mathrm{~nm}$ [Putti et al. (2010)] and following calibration explained in chapter 2, the "true" critical current, $j_{c}$ can be derived. Figure 5-4 shows the critical current as a function of temperature for different magnetic fields. For stronger fields, the critical 
current becomes less temperature-dependent, similarly to the case for LiFeAs.

Finally, Figure 5-5 shows critical current density, $j_{c}$, as a function of magnetic field at different temperatures. The curves are likely to be non-monotonic at high $\mathrm{T}$.

Maximum critical current values, $j_{c}(2 K)=3.3 \times 10^{6} \frac{\mathrm{A}}{\mathrm{cm}^{2}}$.

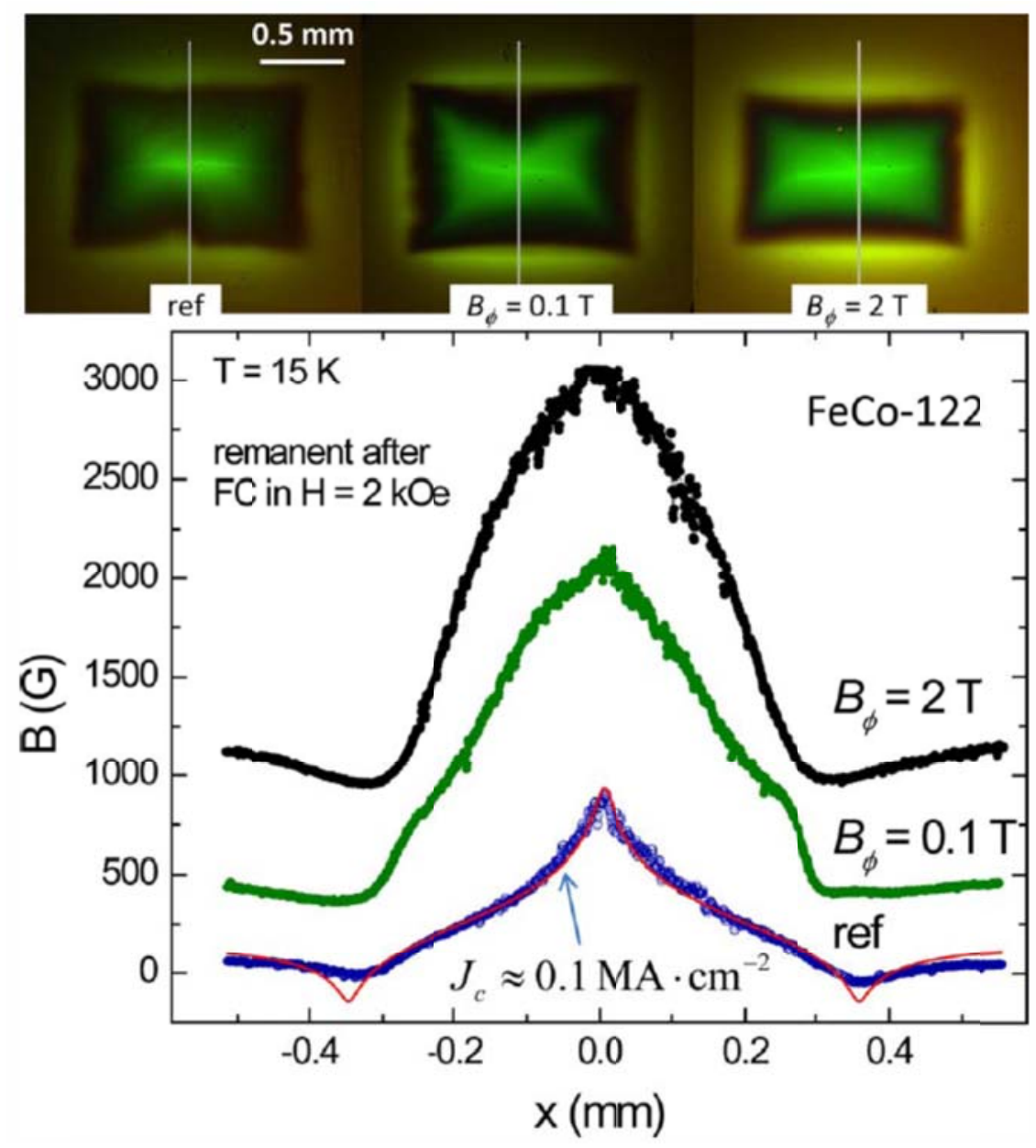

Figure 5-1: Magneto-optic image of the remanent field inside the reference (unirradiated) crystal C0, and irradiated with $\boldsymbol{B}_{\boldsymbol{\phi}}=2 \mathrm{~T}$ crystal, C3. [Prozorov et al. (2010)] 


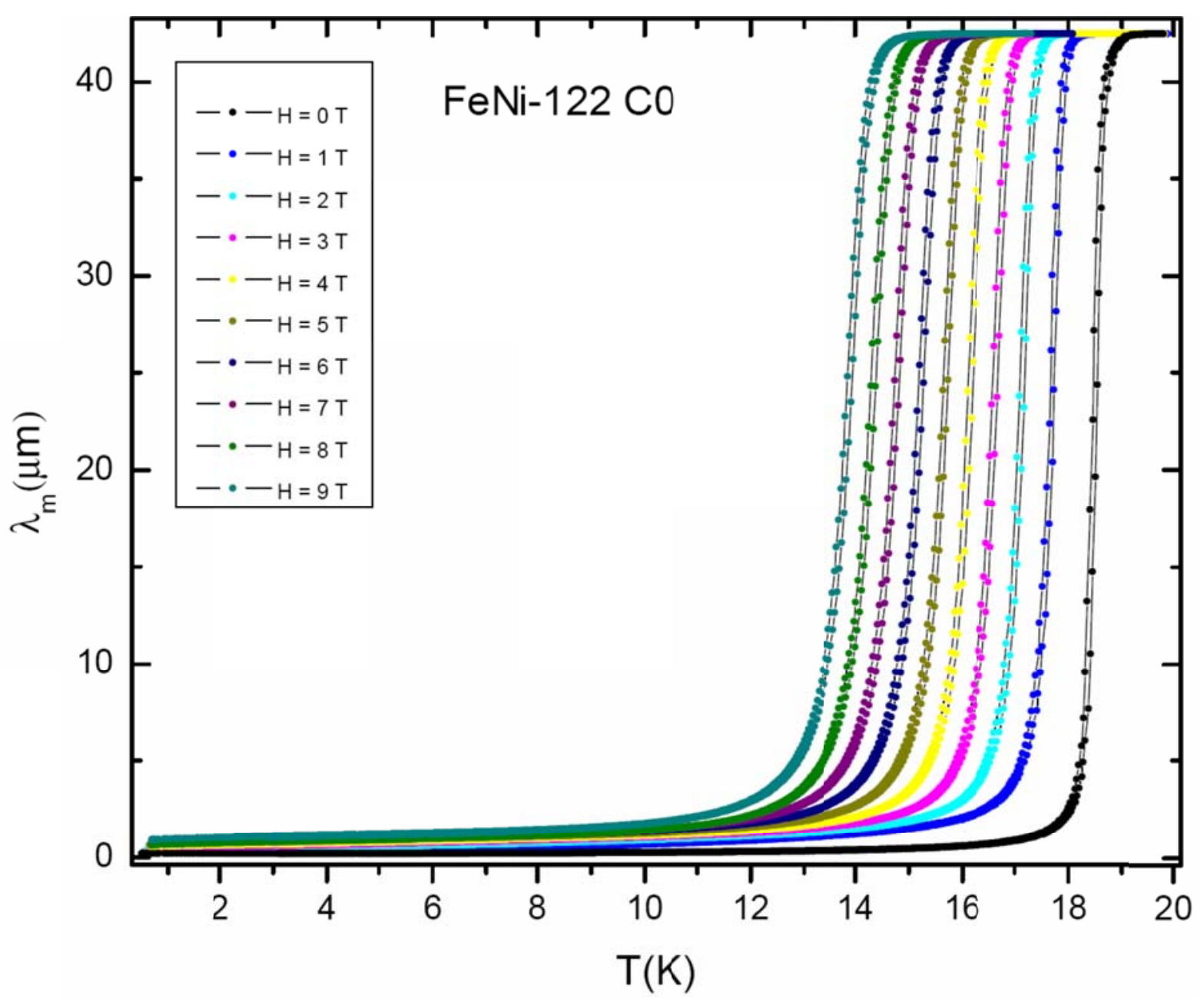

Figure 5-2: Temperature dependence of magnetic penetration depth in optimally doped and unirradiated FeNi-122 crystal measured in ZFC-FC process at different field. $\boldsymbol{T}_{\boldsymbol{c}}=\mathbf{1 8} \mathrm{K}$ in $\mathrm{H}=0 \mathrm{~T}$. 


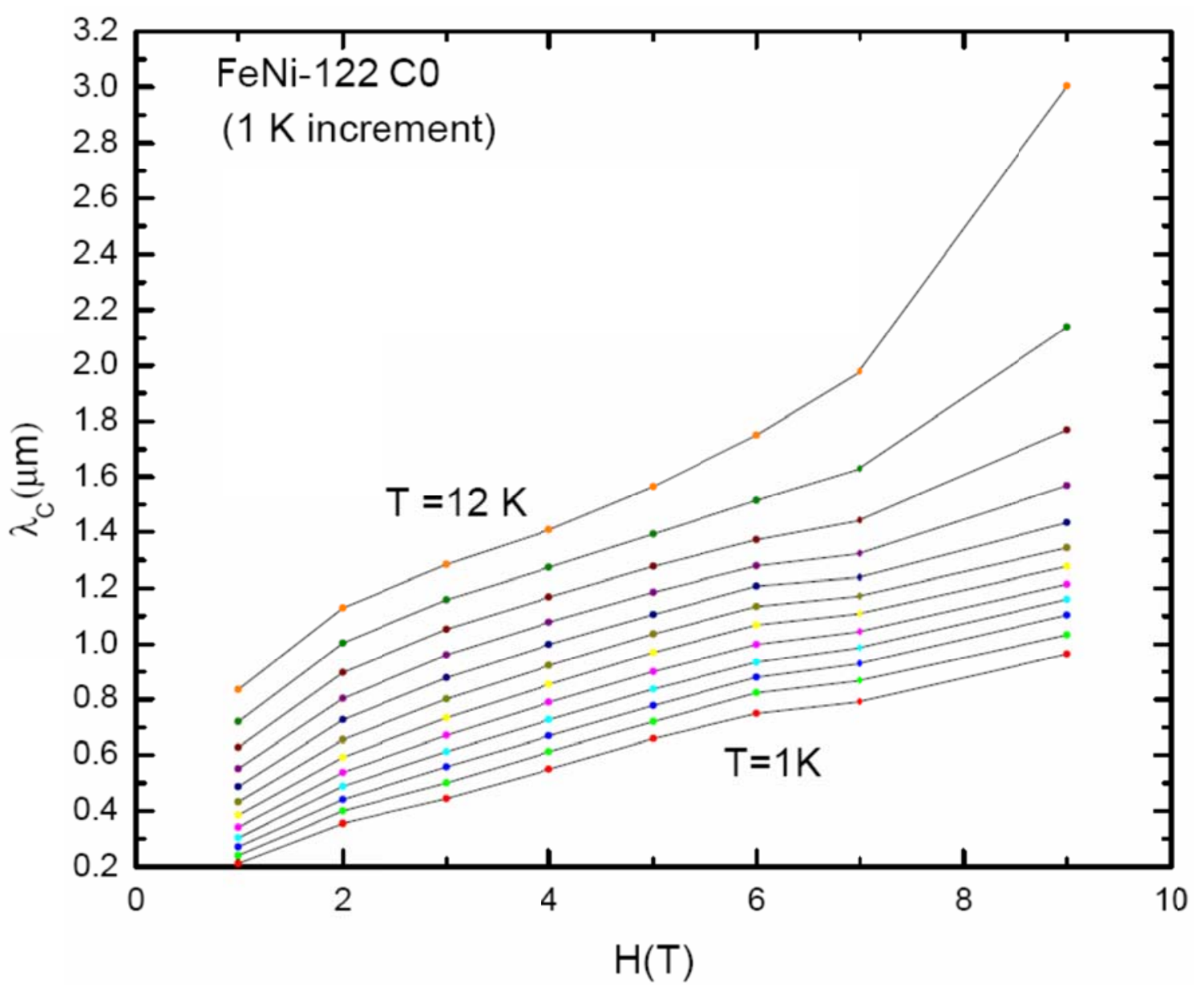

Figure 5-3: Magnetic field dependence of Campbell penetration depth in unirradiated FeNi-122 crystal from ZFC process at different temperatures. 


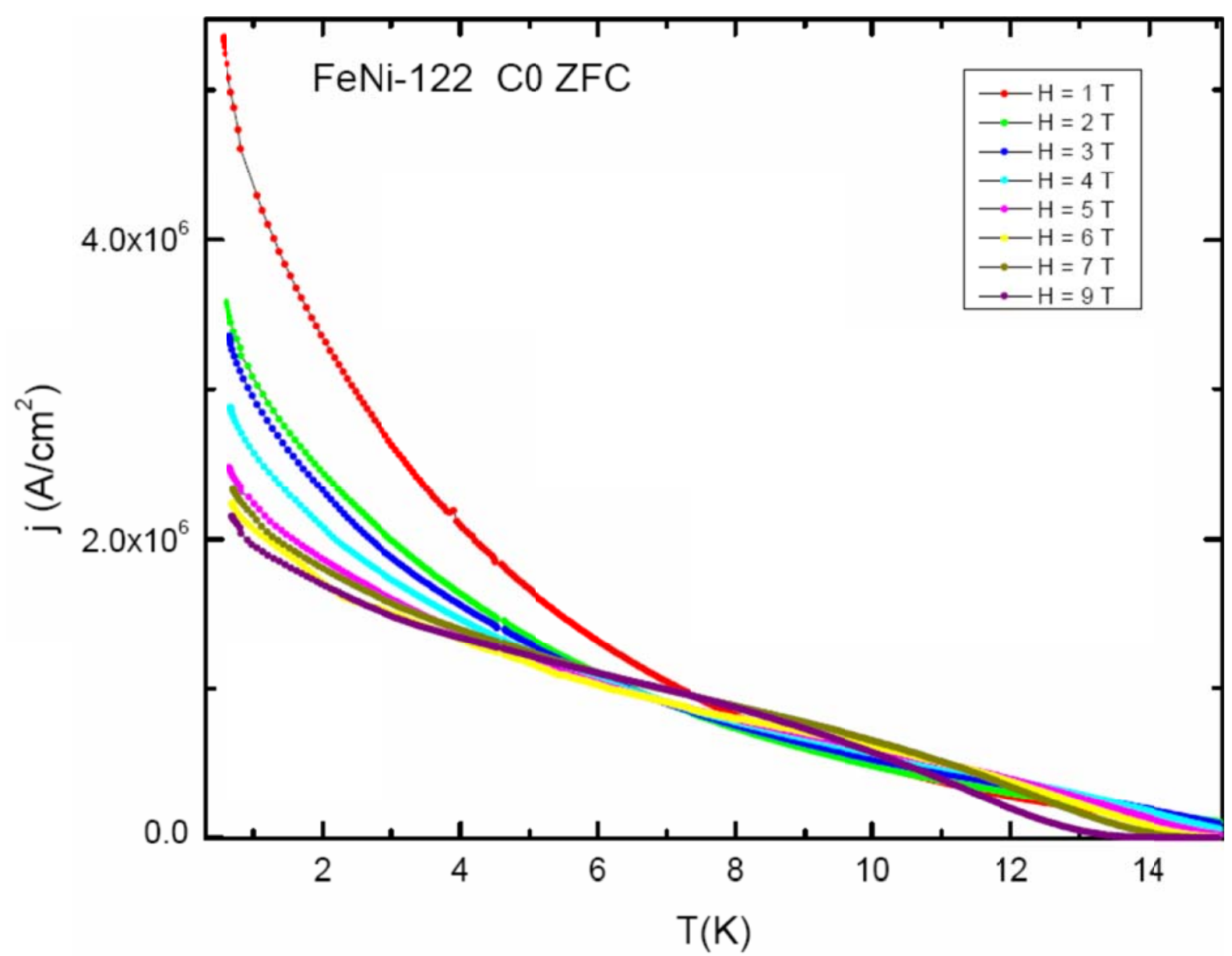

Figure 5-4: Critical current density determined from ZFC Campbell penetration depth as a function of temperature at $\mathrm{H}=1 \mathrm{~T}$ to $9 \mathrm{~T}$. 


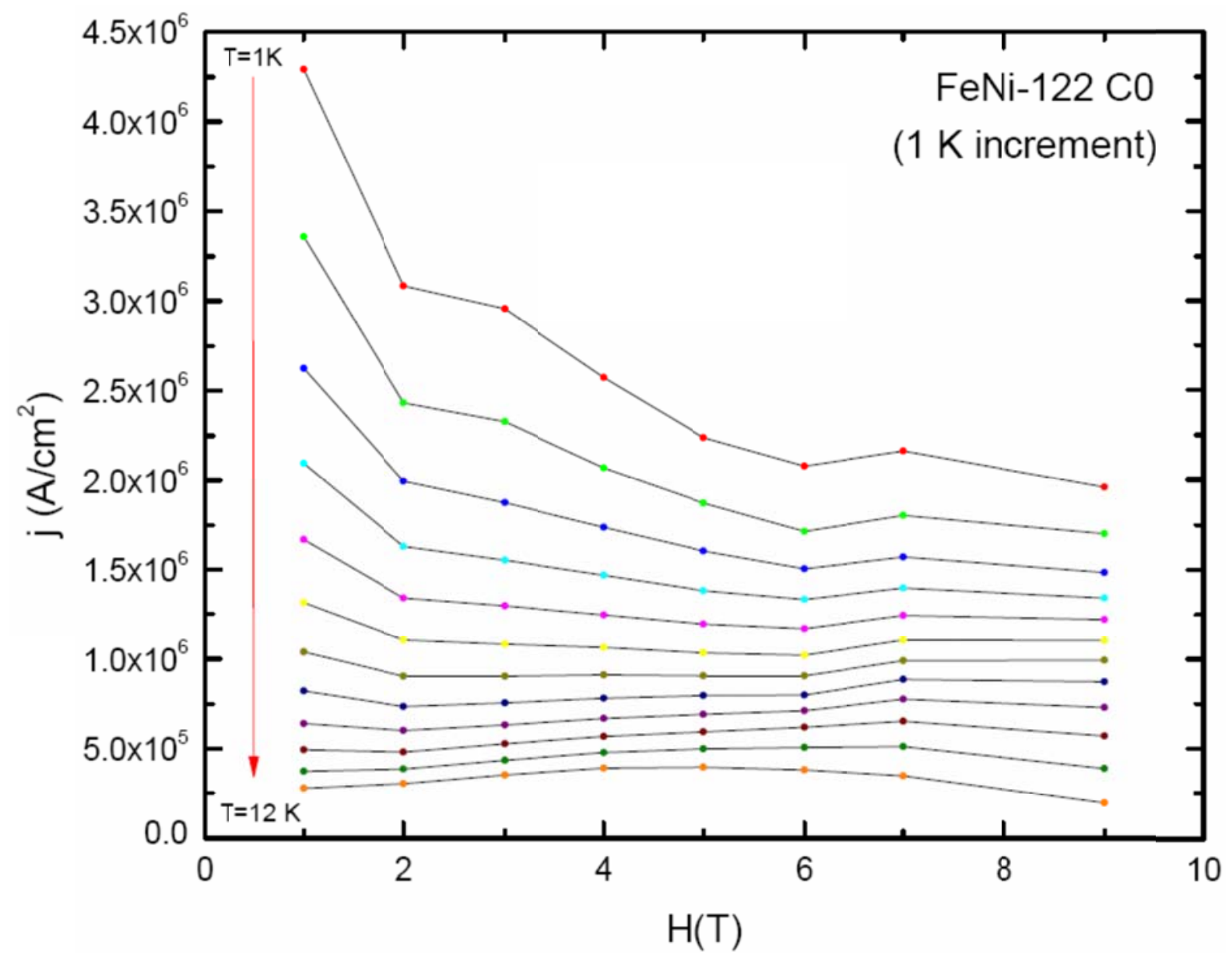

Figure 5-5: Magnetic field dependence of critical current density for FeNi-122 $\mathrm{CO}$ at $\mathrm{T}=1 \mathrm{~K}$ to $\mathrm{T}=12 \mathrm{~K}$. 
Irradiated $\mathrm{Ba}\left(\mathrm{Fe}_{0.954} \mathrm{Ni}_{0.046}\right)_{2} \mathrm{As}_{2}$ and $\mathrm{FeTe}_{0.53} \mathrm{Se}_{0.47}$

Next, we will discuss the results obtained in irradiated FeNi-122 which have been reported that the introduction of defects by heavy-ion irradiation or columnar defects leads to the enhancement of pinning, resulting in higher critical current than unirradiated crystals [Prozorov et al. (2010)]. The procedure for the measurement is ZFC and subsequent with FW cycle process in the same way as described in LiFeAs. Figure 5-6 shows the magnetic penetration depth in irradiation FeNi-122 (C3). Zero- field transition temperature of C3 sample was approximately $T_{c}=18 \mathrm{~K}$ but slightly lower than in C0 sample. No significant hysteresis between ZFC and FC process indicates that the pinning potential has possible parabolic shape. The most remarkable result is that at the transition temperature, there is a paramagnetic uptrend which is magnetic dependent and decays as in the trend of Curie Weiss law. The feature was not observed in the unirradiated sample $\mathrm{C} 0$. It might suggest the defects caused by heavy-ion irradiation are magnetic dependent. This feature also was observed in magnetic penetration depth in the optimal doped $F e T e_{1-x} S e_{x}$ ironchalcogenides superconductors as shown in Figure 5-7. Because of this uptrend feature, further studies are required in order to appropriately calibrate the magnetic dependent Campbell penetration depth. In addition, we have the evidence that the physical properties of irradiated sample might change over time. Figure 5-8 shows the London penetration depth measured at different time. The transition temperatures of all our three measurements were slightly higher than the transition in the experiment conducted over one year ago. 


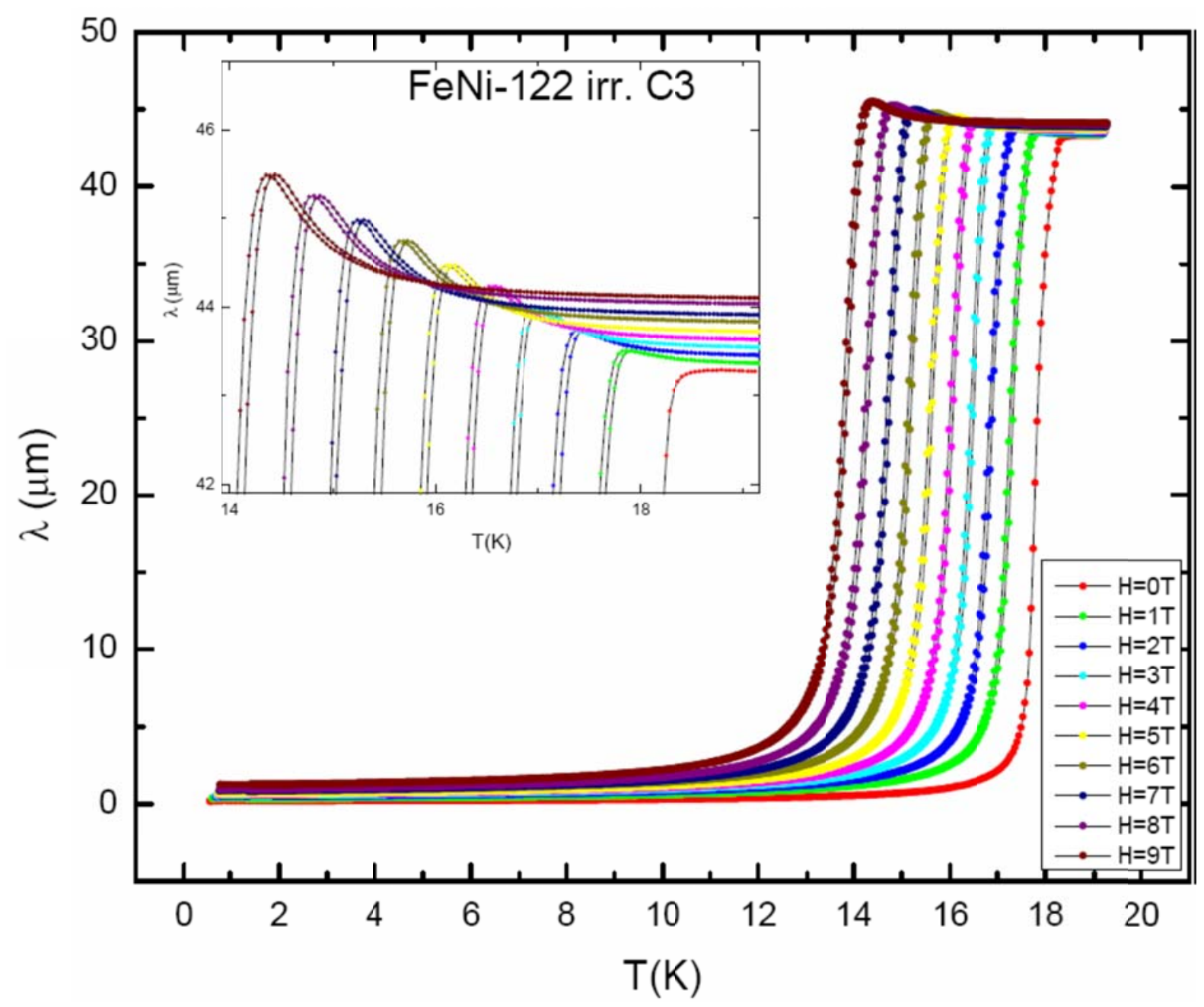

Figure 5-6: Temperature dependence of magnetic penetration depth measured in a ZFC-FC process at different fields in irradiated FeNi122, C3 crystal. Inset shows the overshoot feature observed in the magnetic field. 


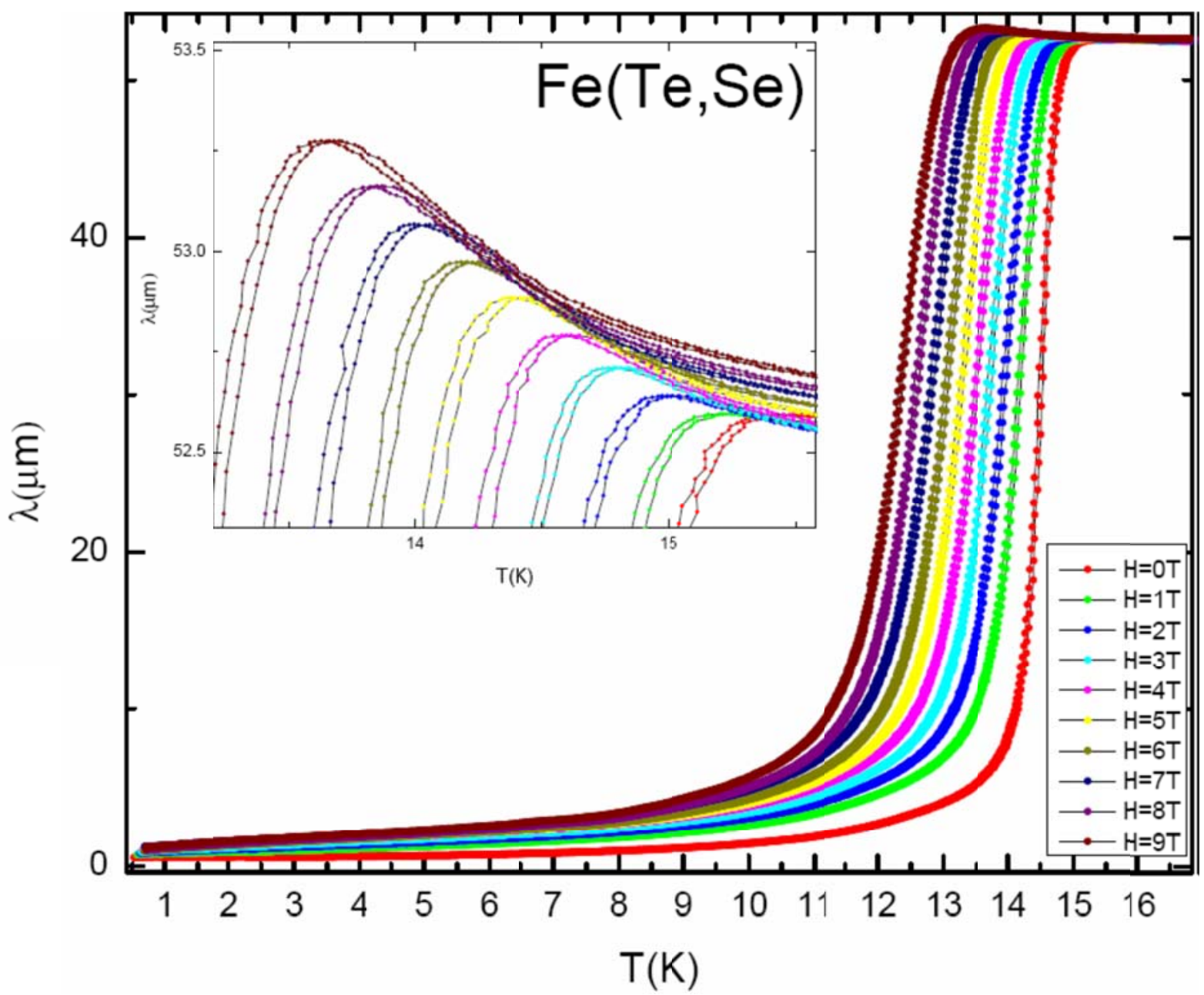

Figure 5-7: The magnetic penetration depth as function of temperature in optimal doped $\mathrm{Fe}(\mathrm{Te}, \mathrm{Se})$ at different fields. Inset shows the overshoot feature similar to be observed in in irradiated FeNi122, C3. 


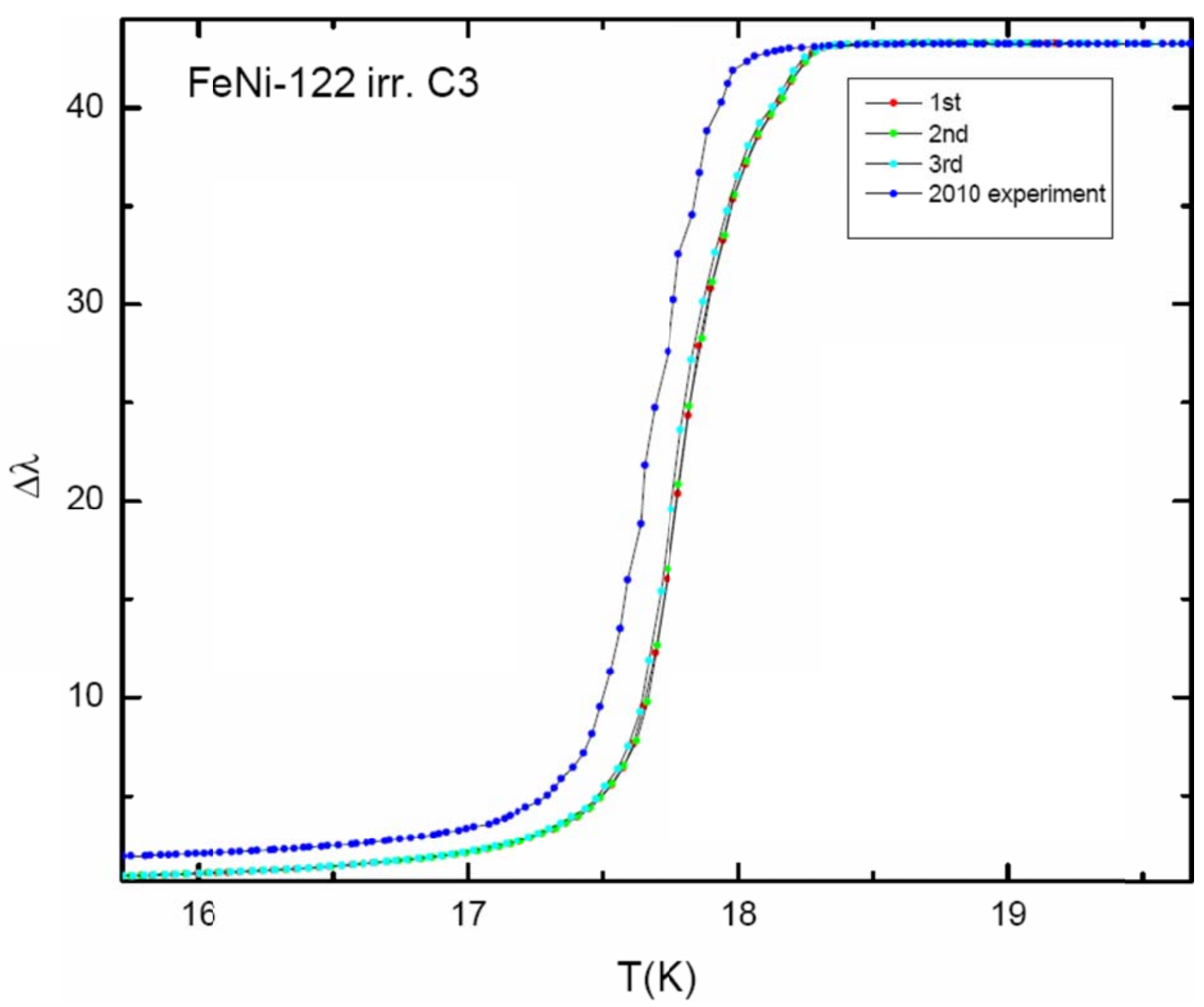

Figure 5-8: The London penetration depths were measured at the different time frame. The blue curve was measurement in 2010 , one year earlier than the rest of the experiment. 


\section{$\mathrm{Ba}_{0.6} \mathrm{~K}_{0.4} \mathrm{Fe}_{2} \mathrm{As}_{2}$ and $\mathrm{BaFe}_{2}\left(\mathrm{As}_{0.7} \mathrm{P}_{0.3}\right)_{2}$}

Now, let us discuss the results from the optimal hole-doped $\left(B a_{1-x} K_{x}\right) F_{2} A s_{2}$ (BaK122). Our first interest in BaK122 is that it has significantly high $T_{c}=38 \mathrm{~K}$ [Rotter, Tegel, and Johrendt (2008)]. The critical current had been reported to be about $4.7 \times 10^{6} \mathrm{~A} / \mathrm{cm}^{2}$ at $\mathrm{T}=2 \mathrm{~K}$ and fishtail effect have been observed [Yang et al. (2008a)]

Figure 5-9 shows the magnetic penetration depth in BaK122 by initially zero field cooling-field warming (ZFC-FW) process and then immediately following by field cooling $(\mathrm{FC})$ process. At low temperature, magnetic penetration depth in some applied magnetic field crossed with the London penetration depth which contradicts with eq. $\lambda^{2}=\lambda_{L}^{2}+\lambda_{C}^{2}$. The similar situation had been observed in optimal doped $\mathrm{BaFe}_{2}\left(A s_{1-x} P_{x}\right)_{2} \quad(\mathrm{BaP} 122)$. The magnetic penetration depth in $\mathrm{BaP} 122$ is presented in Figure 5-10. One possible explanation for crossing between magnetic penetration depths is the anomalous Meissner effect in $\mathrm{BaFe}_{2} \mathrm{As}_{2}$ superconductors reported by [Prozorov 2010b]. This unusual effect proposed that $\lambda(T=0, H=0)>$ $\lambda(T=0, H)$ due to the magnetization gradually becoming more negative as the applied magnetic field increase, and finally it exceeds the thermodynamic critical field. This uncommon effect, calls for further studies in order to appropriately extract the critical current from the Campbell penetration depth. 


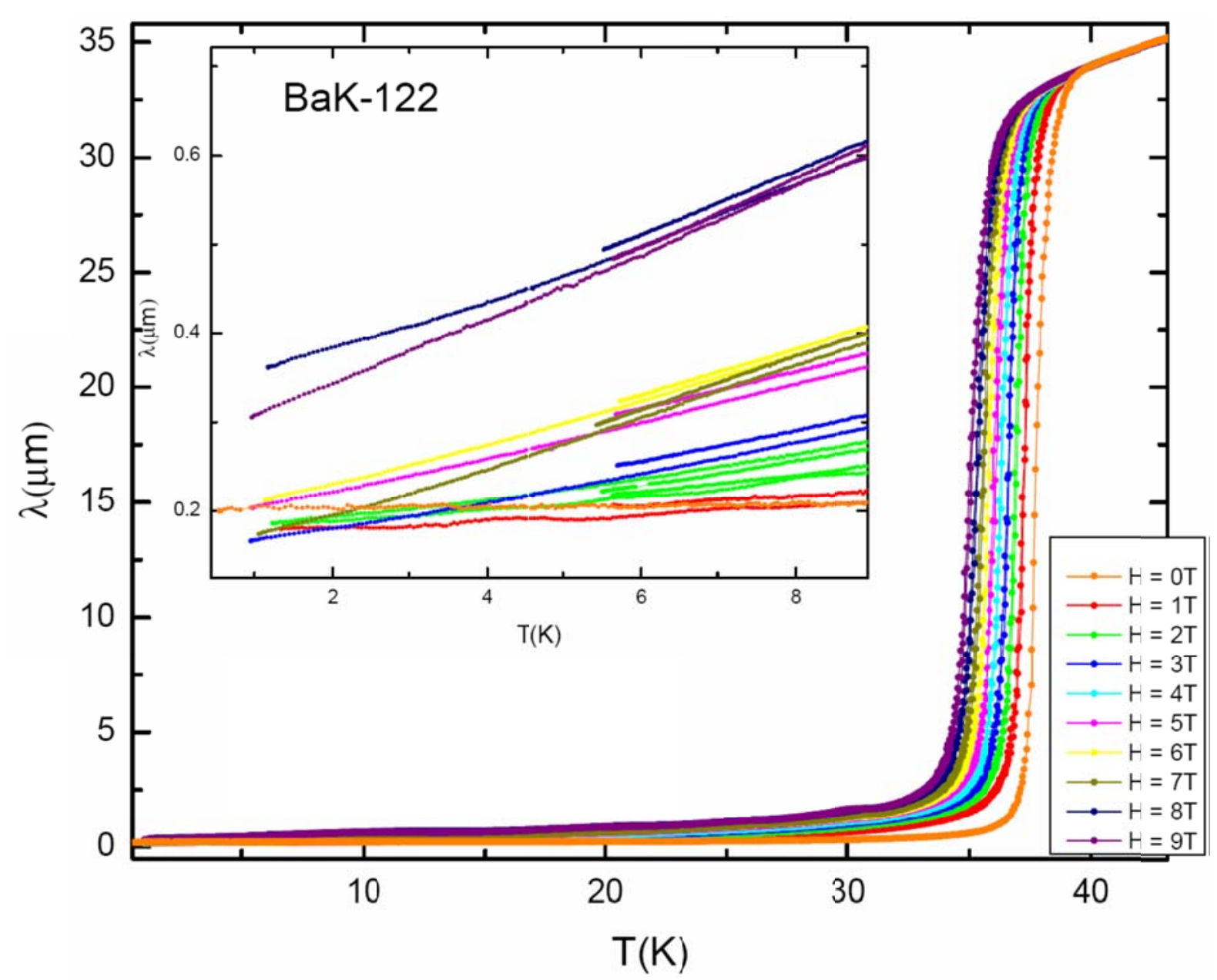

Figure 5-9: Magnetic penetration depth measured in a ZFC-FC process at different field as a function of temperature in BaK122 crystal. Inset shows the magnetic penetration depth at low temperature. Some of magnetic penetration depth crossed with the London penetration depth $(\mathrm{H}=\mathrm{OT})$. 


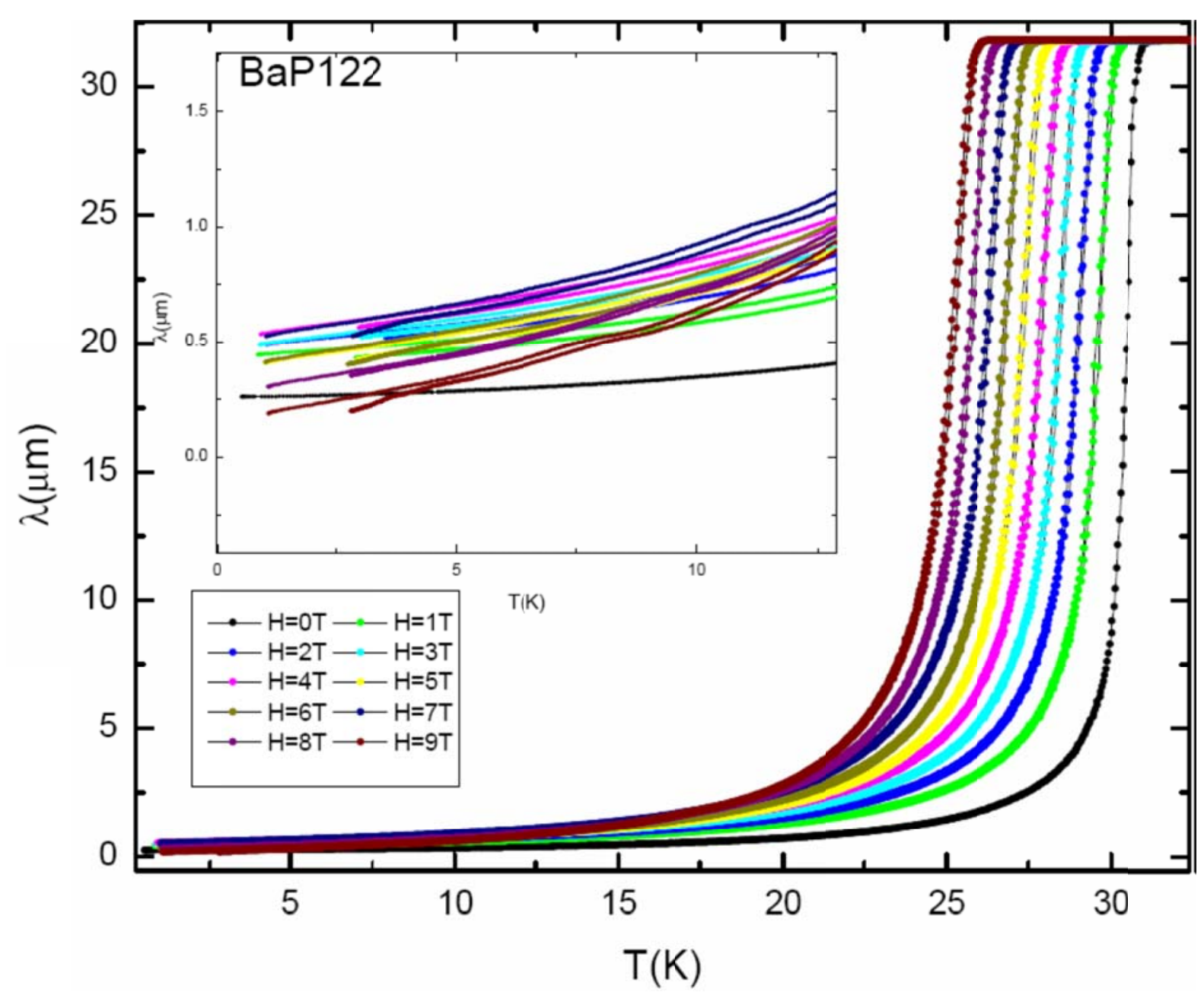

Figure 5-10: Magnetic penetration depth measured in a ZFC-FC process at different field in BaP122 crystal. Inset shows the magnetic penetration depth at low temperature. Similar to BaK122, some of magnetic penetration depth crossed with the London penetration depth $(\mathrm{H}=\mathrm{OT})$. 


\section{Chapter 6}

\section{Campbell penetration depth in other superconductors}

In this chapter, we report the measurements of the Campbell penetration depth and the critical current density as a function of an applied field and temperature in conventional type II superconductor, niobium $(\mathrm{Nb})$ foil and unconventional noncentrosymmetric superconductor, $\mathrm{Mo}_{3} \mathrm{Al}_{2} \mathrm{C}$. The purpose of this chapter is to use Campbell penetration depth to investigate non-Fe-based superconductors and to compare the result with other conventional methods.

\section{High purity $\mathrm{Nb}$ foil}

We have measured the magnetic penetration depth in the mixed state of the high purity niobium foil. The $0.7 \times 0.7 \times 0.05 \mathrm{~mm}^{3}$ sample was placed into the cryostat for the magnetic measurements. The setup of the experiment apparatus was described in Chapter 3. The shift of the resonant frequency (in cgs units) is given by $\Delta f(T)=-G\left[\left(\frac{\lambda}{R}\right) \tanh \left(\frac{R}{\lambda}\right)-1\right]$, where $R$ is the characteristic sample size, $G=$ $\frac{f_{0} V_{S}}{2 V_{c}(1-N)}$ is a calibration constant, $\mathrm{N}$ is the demagnetization factor, $V_{S}$ is the sample volume and $V_{c}$ is the coil volume. The skin depth is given by $(T)=\frac{c}{2 \pi} \sqrt{\frac{\rho}{f}}$. In the case $\mathrm{T}>\mathrm{T}_{\mathrm{C}}$, the shift of frequency becomes $\Delta f(T)=G\left[1-\operatorname{Re}\left\{\frac{\tanh (\alpha R)}{\alpha R}\right\}\right], \alpha=\frac{1-i}{\delta}$ or 
$\delta=2 \lambda \quad$ [Hardy et al. (1993)]. Figure 6-1 shows magnetic penetration depth measured upon warming after sample was cooled in applied fields (field cooling process).

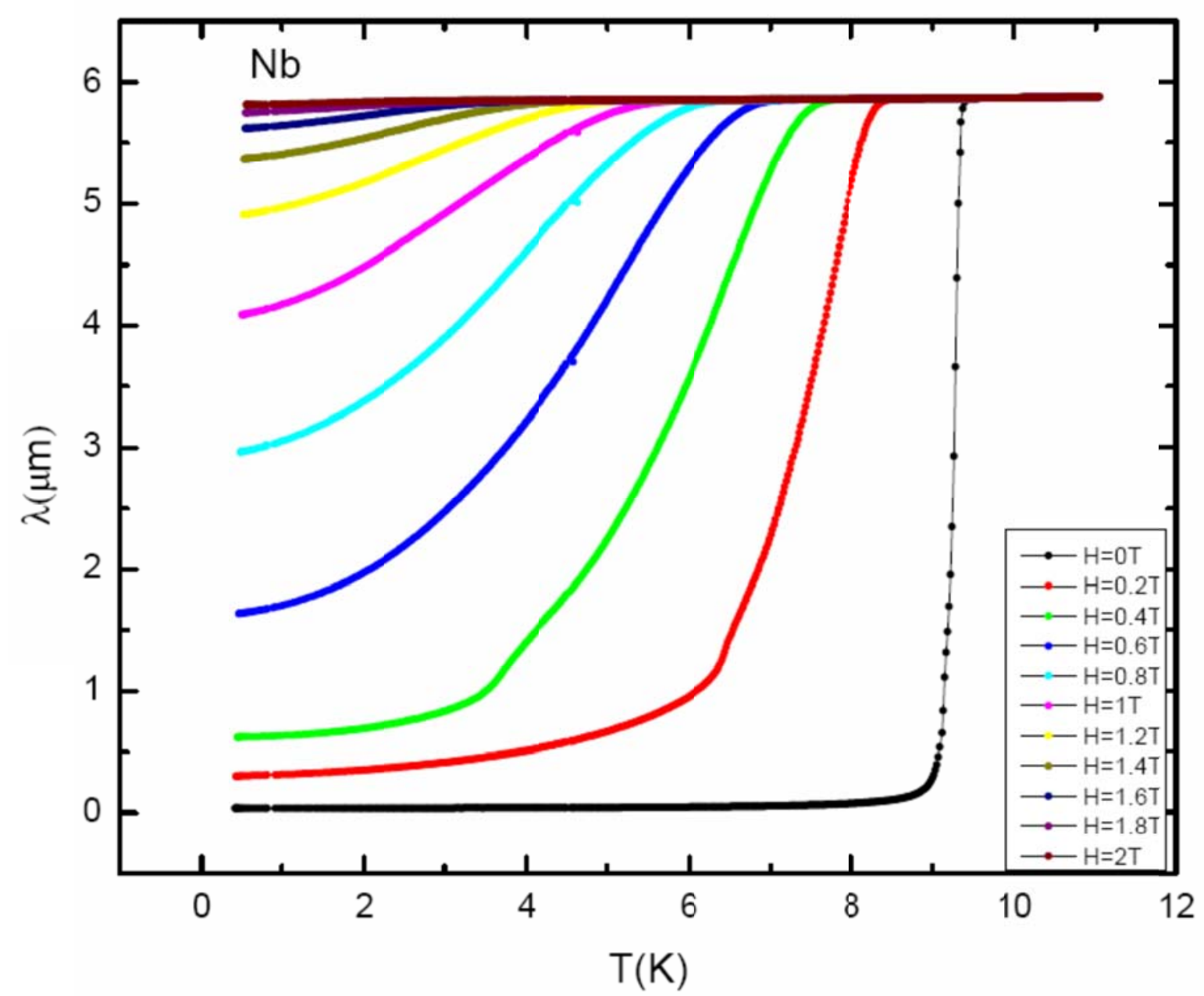

Figure 6-1: Magnetic penetration depth measured in a FC process at different fields.

From the measured penetration depth in zero field above $T_{c}, \lambda\left(T>T_{c}\right)=$ $5.87 \mu m$, the calculated skin depth is equal to $11.74 \mu m$ thus the resitivity, $\rho=$ $0.77 \mu \Omega \mathrm{cm}$. The resitivity is given by the Drude theory as $\rho=m v_{F} /\left(n e^{2} l\right)$ where $\mathrm{m}$ 
is the effecive mass of conduction electrons and $e$ is the electron charge. For niobium, the Fermi velocity, $v_{F}=1.37 \times 10^{6} \mathrm{~ms}^{-1}$ and the denesity of conduction electron, $n=5.56 \times 10^{28} \mathrm{~m}^{-3}$ [Ashcroft and Mermin (1976)]. Hence using the Drude formula the mean free path, $l=112 \mathrm{~nm}$. Since the means free path is greater than the coherence length, $\xi=38 \mathrm{~nm}$ [Maxfield and McLean (1965)], our material is in the clean limit. We determine the Campbell penetration depth via, $\lambda_{C}=\sqrt{\lambda^{2}-\lambda_{L}^{2}}$ where the London penetration depth, $\lambda_{L}$, is the measured penetration depth in zero field. At zero temperature, $\lambda_{L}=39 \mathrm{~nm}$ [Maxfield and McLean (1965)]. We extract the critical current density from the Campbell penetration depth as $\frac{4 \pi}{c} j_{c}=\frac{r_{p} \phi_{0}}{\lambda_{C}^{2}}$, where we assumed the radius of the pinning potential to be the coherence length, $r_{p} \simeq \xi \simeq 38 \mathrm{~nm}$. Figure 6-2 shows $j_{c}$ as a function of temperature at different magnetic fields determined in FC process.

\section{Conclusion}

Our result shows that the maximum critical current values of $j_{c}(2 \mathrm{~K}) \approx 4.94 \times$ $10^{6} \mathrm{~A} / \mathrm{cm}^{2}$ and $j_{c}(4.2 \mathrm{~K}) \approx 2.12 \times 10^{6} \mathrm{~A} / \mathrm{cm}^{2}$. For comparison, the critical currents in $\mathrm{Nb}$-strips at $4.2 \mathrm{~K}$ have been reported between $3-7 \times 10^{6} \mathrm{~A} / \mathrm{cm}^{2}$ depending on substrate temperatures during the film deposition [Huebener et al. (1975)]. From the magnetic penetration depth, no clear-cut temperature transition has been observed when the external magnetic fields are applied. This coincides with the sudden changes in the magnetic-flux distribution due to rapid redistribution of Abrikosov vortices in Nb foils reported in [Prozorov, Shantsev and Mints (2006)]. 


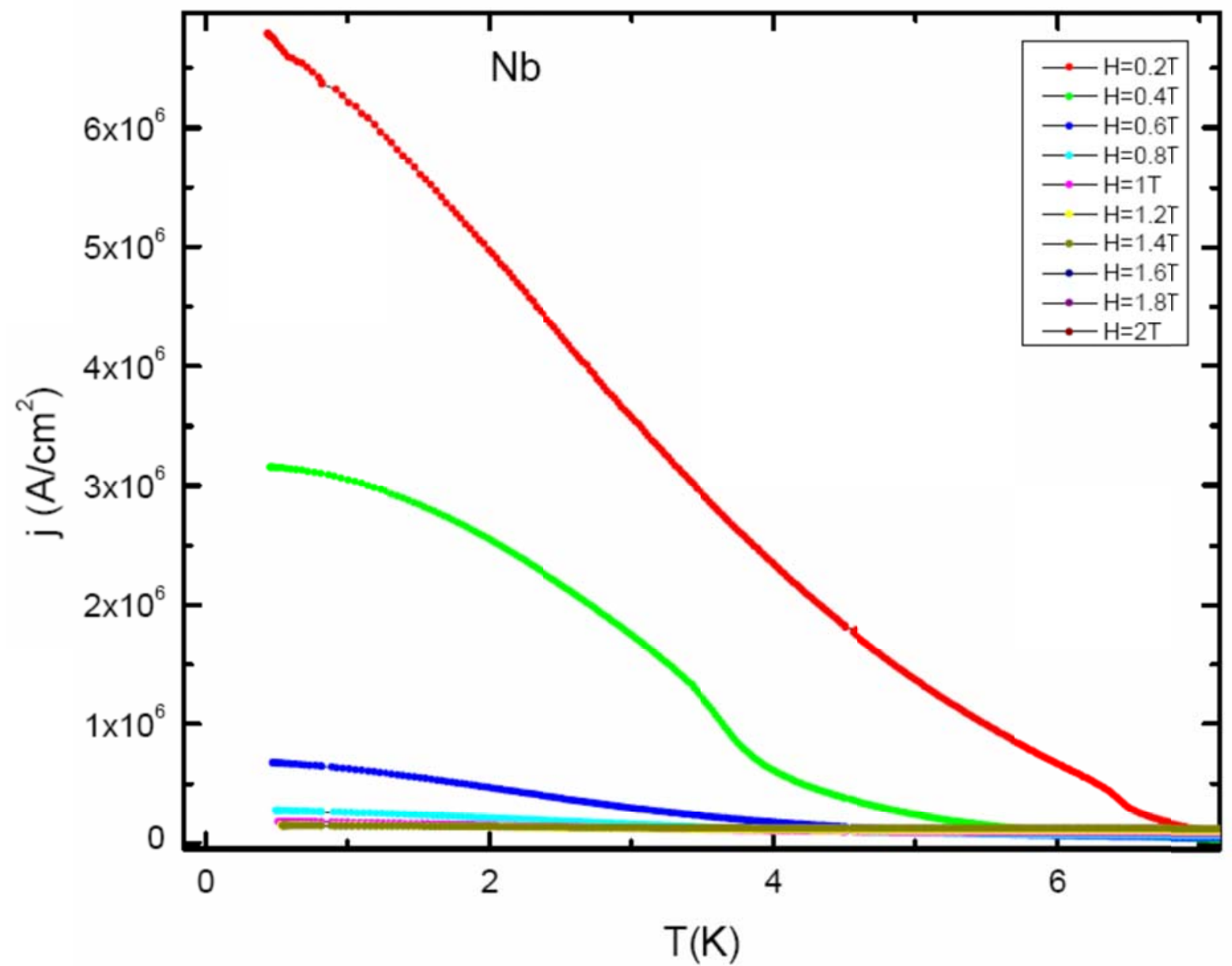

Figure 6-2: The critical current density, $\boldsymbol{j}_{\boldsymbol{c}}$, determined from the FC experiment at indicated values 
$\mathrm{Mo}_{3} \mathrm{Al}_{2} \mathrm{C}$

Superconducting $\mathrm{Mo}_{3} \mathrm{Al}_{2} \mathrm{C}$ was discovered in 1963 [Jeitschko, Nowotny and Benesovsky (1963a,1963b); Johnston et al. (1964)]. The unique feature of $\mathrm{Mo}_{3} \mathrm{Al}_{2} \mathrm{C}$ is the noncentrosymmetric $\beta$-Mn structure, which does not have a center of inversion. Figure 6-3 shows the crystal structure of $\mathrm{Mo}_{3} \mathrm{Al}_{2} \mathrm{C}$. The polycrystalline sample was prepared by arc and RF melting. More details about the preparation method and physical parameters such as penetration depth $\lambda(0)$, coherence length $\xi(0)$ can be found in [Karki et al. (2010); Bauer et al.(2010)].

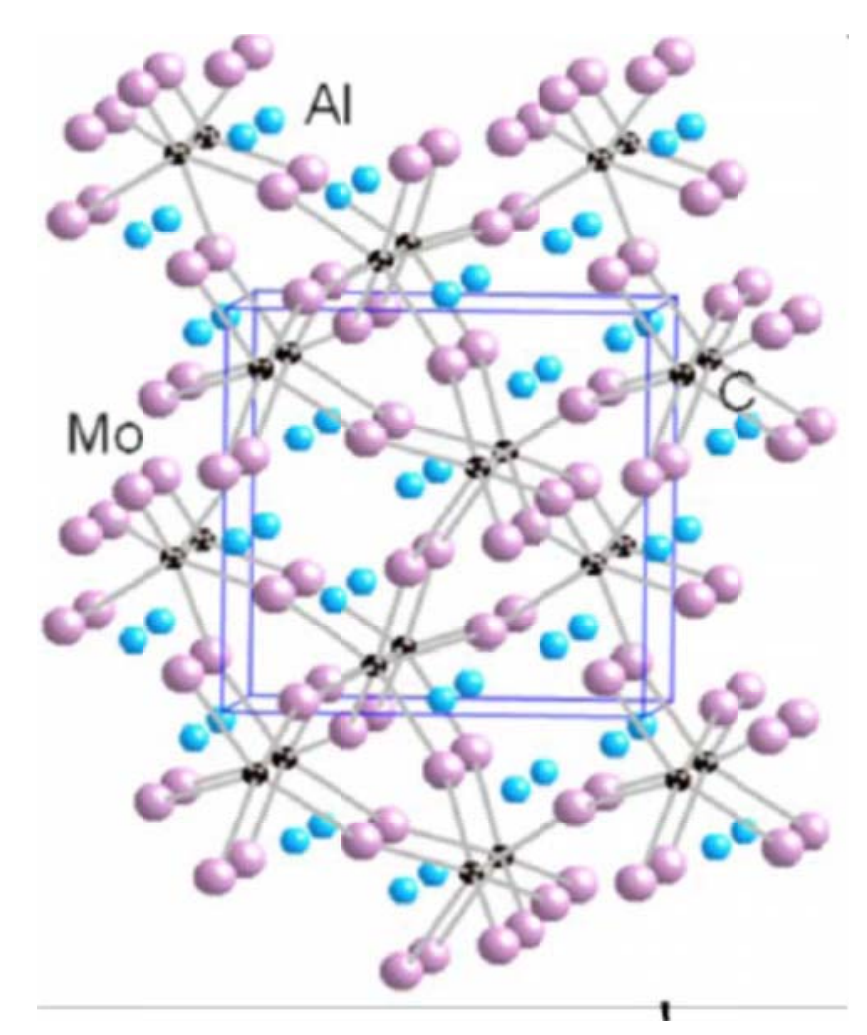

Figure 6-3: The crystal structure of $\mathrm{Mo}_{3} \mathrm{Al}_{2} \mathrm{C}$ [Karki et al. (2010)] 
In this part, we report the measurement of the magnetic penetration depth and the estimated critical current from Campbell penetration depth. A $0.8 \times 0.5 \times$ $0.3 \mathrm{~mm}^{3}$ polycrystalline sample was cut and polished for TDR measurements. The magnetic penetration depth was measured in both zero field cooled (ZFC) and field cooled (FC) procedures.

\section{Results and conclusion}

Figure 6-4 shows the magnetic penetration depth, $\lambda_{m}$, measured as function of temperature at different magnetic fields. The main superconducting transition temperature was observed at $9 \mathrm{~K}$ in zero field. Our measurements show very strong hysteresis and initially rise at $\lambda_{m} \approx 20 \mu \mathrm{m}$ in high magnetic fields. The hysteresis between ZFC and FC is much larger than that observed in Fe-based superconductors as discussed earlier. In weak magnetic fields, two field-dependent superconducting phase transitions were observed as shown in Figure 6-5. The inset in Figure 6-5 shows that both transition temperatures are inversely proportional to the applied magnetic fields. However, the origins of these transitions have not yet been fully understood yet. 


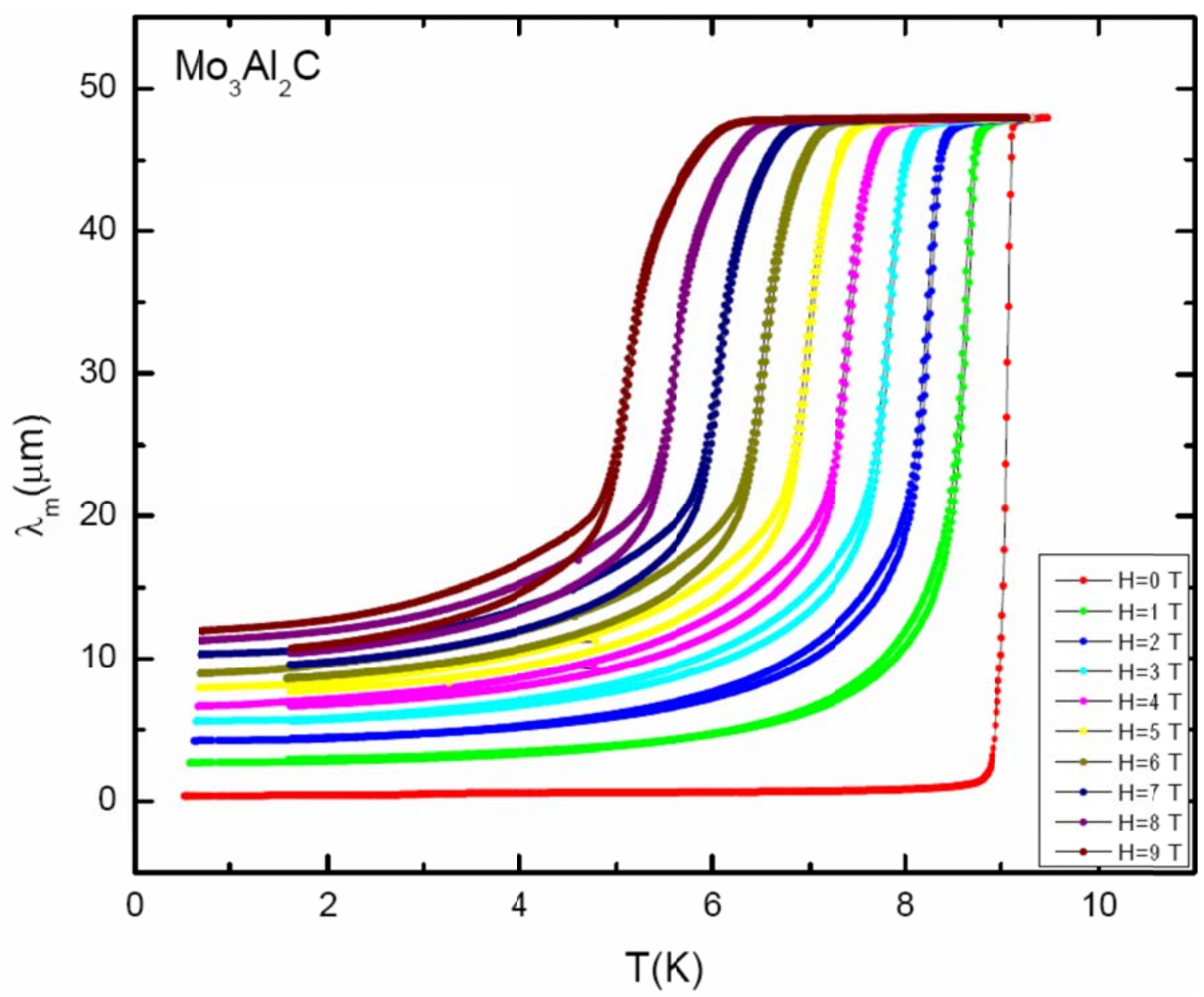

Figure 6-4: Magnetic penetration depth measured in a ZFC process, and FC processes at different fields. 


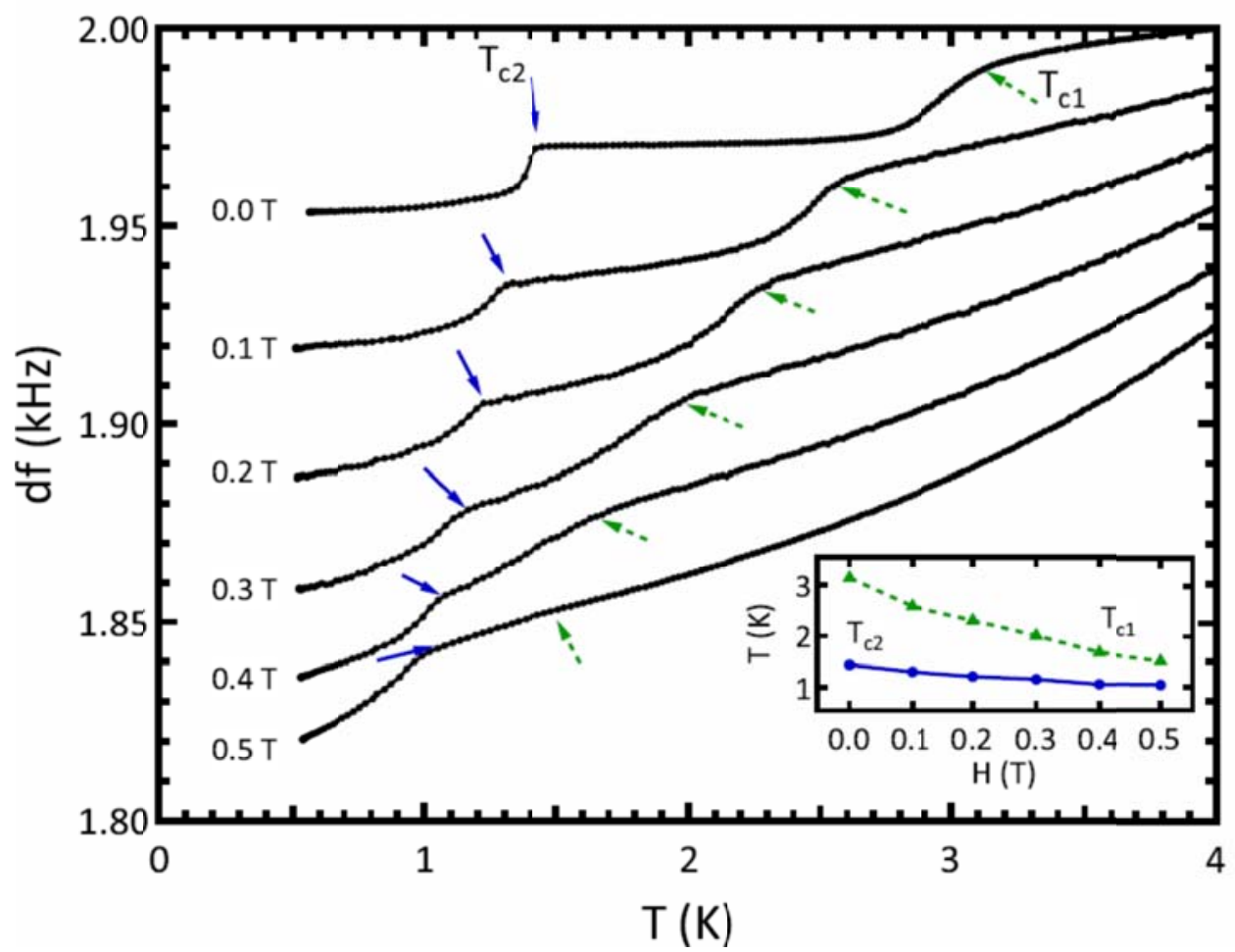

Figure 6-5: Two field-dependent transitions were observed for low applied magnetic fields. The inset shows both transition temperatures as function of the applied magnetic fields.

Next, the Campbell penetration depth is determined via, $\lambda_{C}=\sqrt{\lambda_{m}^{2}-\lambda_{L}^{2}}$ as shown in Figure 6-6. Obviously, $\lambda_{C, Z F C}>\lambda_{C, F C}$ therefore the pinning potential $V(r)$ is non-parabolic for $\mathrm{Mo}_{3} \mathrm{Al}_{2} \mathrm{C}$. With the coherence length, $\xi(0)=423 \mathrm{~nm}$ and the London penetration depth, $\lambda(0)=3775 \AA$ [Karki et al. (2010)], we determined the critical current density, $j_{C}$, from the Campbell penetration depth. Figure 6-7 shows $j_{C}$ as a function of temperature at different magnetic fields as determined after a ZFC process (top frame) and FC process (bottom frame). In both cases, the curves are monotonic in temperature. $j_{C, Z F C}>j_{C, F C}$ in the presence of Bean current due to a macroscopic vortex density gradient in the ZFC process. 


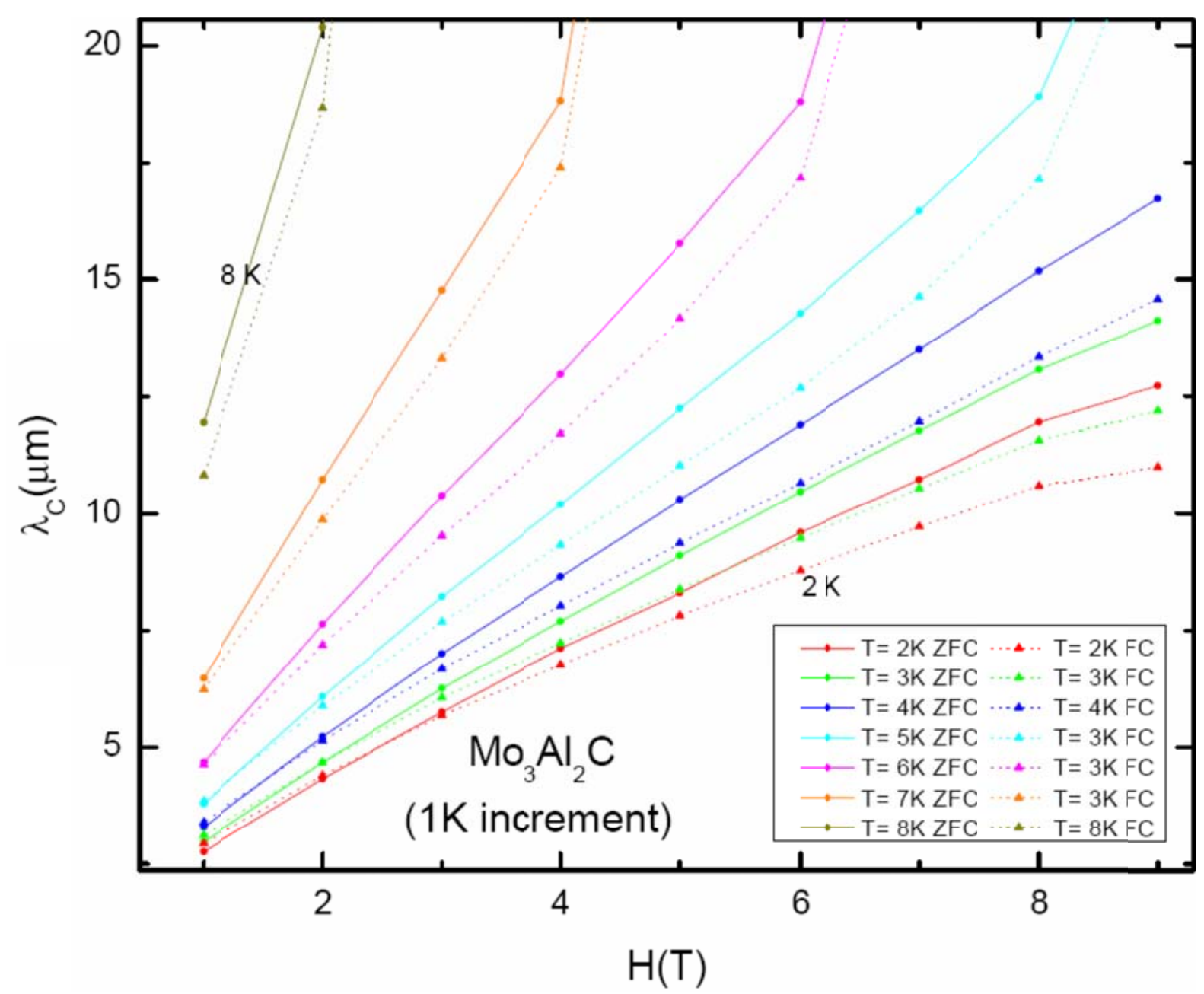

Figure 6-6: Campbell penetration depth as function of magnetic field at different temperatures extracted from the data of Figure 6-4. Solid lines are ZFC and dashed lines are FC data. 


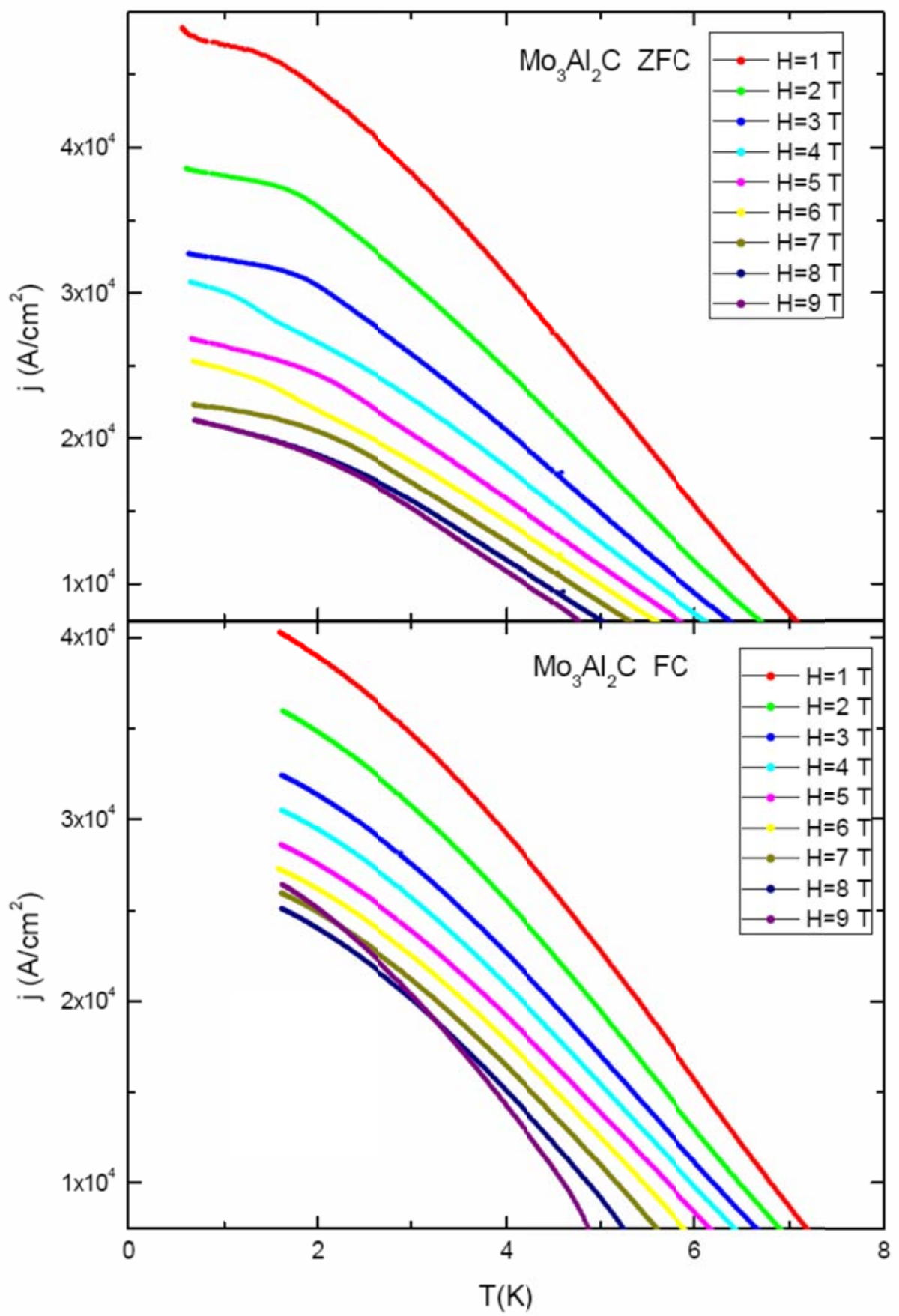

Figure 6-7: Critical current density, $\boldsymbol{j}_{\boldsymbol{c}}$, determined from the ZFC (top frame) and FC (bottom frame) experiments at various values of the applied external magnetic fields. 
Finally, Figure 6-8 shows the critical current density, $j_{C}$, determined from ZFC Campbell penetration depth and FC Campbell penetration depth as a function of magnetic field at different temperatures. In both cases, the curves are rather monotonic; and no clear fishtail signature is observed. The values of $j_{C}(2 K) \approx 4.4 \times$ $10^{4} \mathrm{~A} / \mathrm{cm}^{2}$ provide an upper estimate of the critical current of $\mathrm{Mo}_{3} \mathrm{Al}_{2} \mathrm{C}$.

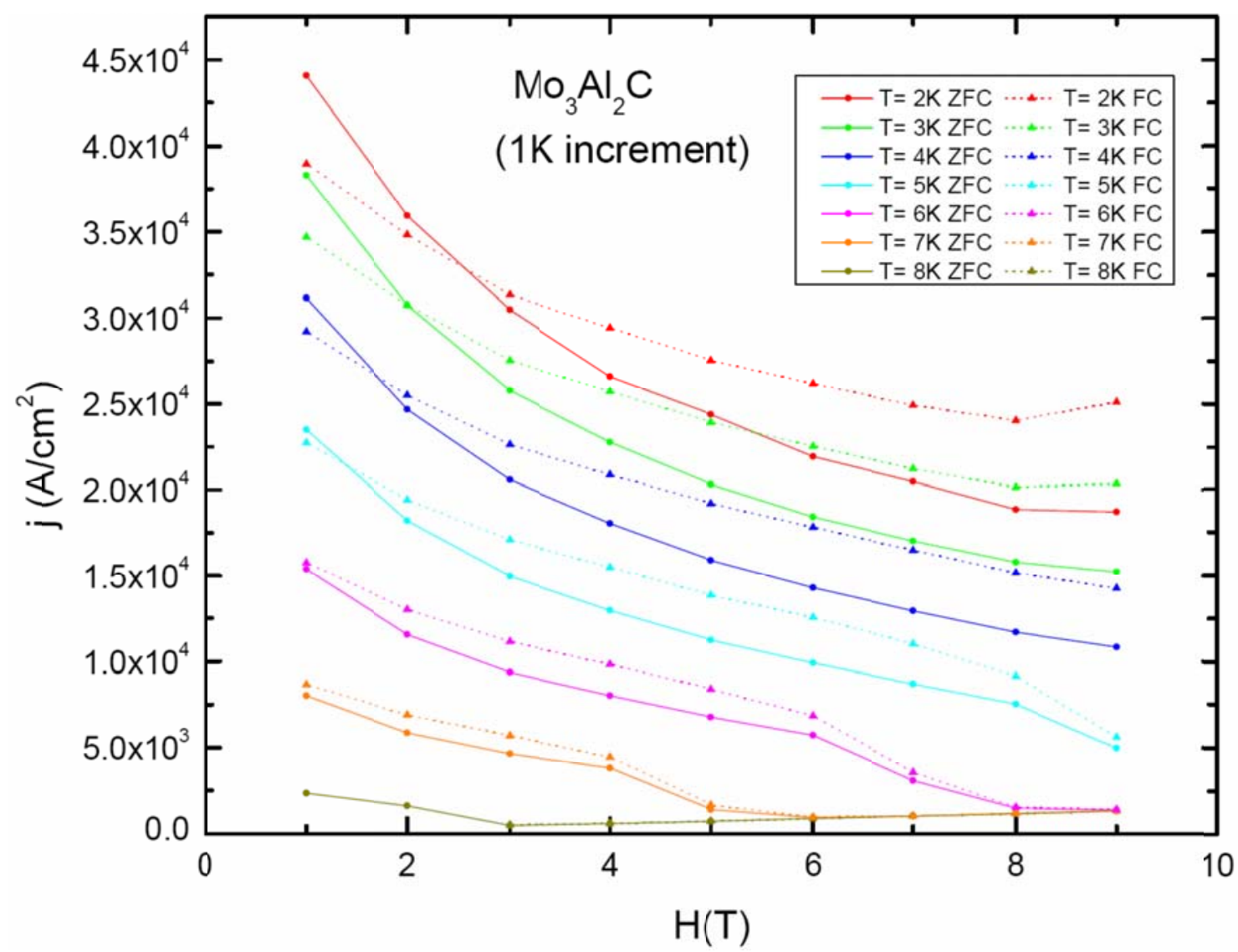

Figure 6-8: Critical current density, $\boldsymbol{j}_{\boldsymbol{c}}$, as function of magnetic field at different temperatures extracted from the Campbell penetration depth. Solid lines are ZFC and dashed lines are FC data. 


\section{Chapter 7}

\section{Conclusion}

Most parts of this thesis show that the Campbell penetration depth is a very useful method for studying vortex properties and understanding microscopic mechanisms of vortex pinning phenomena in superconductors. Analysis of the Campbell penetration depth can provide evidence for magnetic field-dependent properties, the shape of effective pinning potential, and the theoretical critical current density. This critical current density is different from the relaxed persistent current density obtained from conventional measurements which usually is underestimated due to significant magnetic relaxation.

We find evidence that the vortex properties in Fe-based superconductors are remarkably similar to high-Tc cuprates materials regardless of being quite different in terms of their paring and gap structure. In LiFeAs, we show that the fishtail effect has a dynamic origin and the magnetic relaxation is field-dependent due to the fielddependence of the Labusch parameter, $\alpha$. Similarly, $\mathrm{Ba}\left(\mathrm{Fe}_{0.954} \mathrm{Ni}_{0.046}\right)_{2} \mathrm{As}_{2}(\mathrm{FeNi122})$ exhibits a high critical current density similar in magnitude to LiFeAs, however the effective pinning potential is quasi-parabolic. The transition temperature in irradiated FeNi122 is slightly lower than in non-irradiated samples. The defects caused by 
heavy-ion irradiation in FeNi122 are magnetic dependent, as we observed the fielddependent paramagnetic uptrend only in irradiated samples.

In high purity $\mathrm{Nb}$ foil, the critical current density and vortex behaviors, as determined from Campbell penetration depth, coincide with the results from other conventional measurements. This confirms that the results from the Campbell penetration depth are reliable. In the noncentrosymmetric superconductor, $\mathrm{Mo}_{3} \mathrm{Al}_{2} \mathrm{C}$, we observed two superconducting phase transitions in weak applied magnetic fields. $\mathrm{Mo}_{3} \mathrm{Al}_{2} \mathrm{C}$ is a fully-gapped superconductor. However, our measurement of magnetic penetration depth shows very strong hysteresis. This may suggest $\mathrm{Mo}_{3} \mathrm{Al}_{2} \mathrm{C}$ is an unconventional superconductor.

In conclusion, the overall vortex behavior of nearly isotropic, fully-gapped LiFeAs is very similar to highly anisotropic d-wave cuprate superconductors. A very interesting question is: How to reconcile our understanding of the very different electronic properties of Fe-based superconductors (almost isotropic) with those of the cuprates (highly anisotropic) despite their having very similar vortex behavior? This calls for further studies in order to understand the similarities of unconventional superconductivity in cuprates and pnictides. 


\section{Bibliography}

Arfken, G. B. and Weber, H. J. (2005). Mathematical Methods for Physicists.

Elsevier.

Bean, C. P. (1964). Rev. Mod. Phys., 36:31.

Blatter, G., Feigel'man, M. V., Geshkenbein, V. B., Larkin, A. I., and Vinokur, V. M. (1994). Rev. Mod. Phys., 66:1125.

Brandt, E. H. (1986). Phys. Rev. B, 34:6514-6517.

Brandt, E. H. (1991). Phys. Rev. Lett., 67:2219.

Brandt, E. H. (1992). Supercond.Sci. Technol., 5:S25-32.

Brandt, E. H. (1995). Reports on Progress in Physics, 58:1465.

Bud'ko, S. L., Ni, N., and Canfield, P. C., (2009). Phys. Rev. B, 79:220516.

Burlachkov, L., Giller, D., and Prozorov, R. (1998). Phys. Rev. B, 58:15067.

Campbell, A. M. (1969). J. Phys. C: Solid St. Phys.. 2:1492.

Campbell, A. M. and Evetts, J.E. (2001). Adv. in Phys., 50:1249-1449.

Canfield, P. C., Bud'ko, S. L., Ni, N., Yan, J. Q., and A. Kracher, (2009).

Phys. Rev. B, 80:060501. 
Cho, K., Kim, H., Tanatar M. A., Song, Y. J., Kwon, Y. S., Coniglio, W. A., Agosta, C. C., Gurevich, A., and Prozorov, R. (2011). Phys. Rev. B, 83:060502.

Coffey, M. W. and Clem, J. R. (1991). Phys. Rev. Lett., 67:386.

Coffey, M. W. and Clem, J. R. (1992). Phys. Rev. B, 45:10527.

Geshkenbein, V. B. and Larkin, A. I. (1989). Zh. Eksp. Teor. Fiz., 95:1108. [Sov. Phys.JETP, 68:639 (1989)]

Gordon, R. T., Kim, H., Tanatar, M. A., Prozorov. R., and Kogan, V. G. (2010). Phys.Rev. B, 81:180501.

Gordon, R.T., Kim, H., Salovich, N., Giannetta, R. W., Fernandes, R. M., Kogan, V. G., Prozorov, T., Bud'ko, S. L., Canfield, P. C., Tanatar, M. A., and Prozorov R. (2010b). Phys. Rev. B, 82:054507.

Gordon, R. T. (2011). London penetration depth measurements in $B a\left(F e_{1-x} T_{x}\right)_{2} A s_{2}$ ( $T=\mathrm{Co}, \mathrm{Ni}, \mathrm{Ru}, \mathrm{Rh}, \mathrm{Pd}, \mathrm{Pt}, \mathrm{Co}+\mathrm{Cu}$ ) superconductors. PhD dissertation, lowa State University.

Hardy, W. N., Bonn, D. A., Morgan, D. C., Liang, R., and Zhang, K. (1993). Phys. Rev. Lett., 70: 3999.

Inosov, D. S., White, J. S., Evtushinsky, D. V., Morozov, I. V., Cameron, A., Stockert, U., Zabolotnyy, V. B., Kim, T. K., Kordyuk, A. A., Borisenko, S. V., Forgan, E. M., Klingeler, R., Park, J. T., Wurmehl, S., Vasiliev, A. N., Behr, G., Dewhurt, C. D., 
and Hinkov, V. (2010). Phys. Rev.Lett., 104:187001.

Irie, F. and Yamafuji, K. (1967). Theory of Flux Motion in Non-Ideal Type-II

Superconductors. J. Phys. Soc. Jpn. , 23:255.

Kim, H.-J., Liu, Y., Oh, Y. S., Khim, S., Kim, I., Stewart, G. R., and K. H. Kim (2009).

Phys. Rev. B, 79:014514.

Kogan, V. G. (2009). Phys. Rev. B, 80:214532.

Koshelev, A. and Vinokur, V. (1991). Physica C, 173:465.

Labusch, R. (1968). Phys. Rev., 170:470.

Labusch, R. (1969a). Crystal Lattice Defects, 1:1-16.

Labusch, R. (1969b). Phys. Stat. Sol., 32:439.

Lee, B., Khim, S., Kim, J. S., Stewart G. R., and Kim, K. H. (2010). Europhys. Lett., 91: 67002.

Matsushita, T. (2007). Flux Pinning in Superconductors. Springer.

Mikitik, G. P., and Brandt, E. H. (2001). Phys. Rev. B, 64:184514.

Ni, N., Tillman, M. E., Yan, J.-Q., Kracher, A., Hannahs S. T., Bud'ko, S. L., and Canfield, P. C. (2008). Phys. Rev. B, 78:214515.

Pramanik, A. K., Harnagea, L., Nacke, C., Wolter, A. U. B., Wurmehl, S., Kataev, V., and B"uchner, B. (2010). arXiv:1009.4896. Phys. Rev. B, 83:094502 (2011). 
Prommapan, P., Tanatar, M. A., Lee, B., Khim, S., Kim, K. H., and Prozorov, R. (2011) arXiv:1103.1398v1.

Prozorov, R., Shaulov, A., Wolfus, Y., and Yeshurun, Y. (1994). J. Appl. Phys., $76: 7621$.

Prozorov, R., Shaulov, A., Wolfus, Y., and Yeshurun, Y. (1995). Phys. Rev. B, $52: 12541$.

Prozorov, R., Giannetta, R., Carrington, A., and Araujo-Moreira F. (2000). Phys. Rev. B, 62:115.

Prozorov, R., Giannetta, R., Kameda, N., Tamegai, T., Schlueter, J., and Fournier, P. (2003). Phys. Rev. B, 67:1845011.

Prozorov, R., and Giannetta, R. W. (2006). Supercond. Sci. Technol.,19:R41.

Prozorov, R., Vannette, M. D., Samolyuk, G. D., Law, S. A., Bud'ko, S. L., and Canfield, P. C. (2006). Phys. Rev. B, 75:014413.

Prozorov, R., Ni, N., Tanatar, M. A., Kogan, V. G., Gordon, R. T., Martin, C., Blomberg, E. C., Prommapan, P., Yan, J. Q., Bud'ko, S. L., and Canfield, P. C. (2008). Phys. Rev. B, 78:224506/1.

Prozorov, R., Tillman, M. E., Mun, E. D., and Canfield, P. C. (2009). New J. Phys., 11:035004.

Prozorov, R., Tanatar, M. A., Blomberg, E. C., Prommapan, P., Gordon, R. T., 
Ni, N., Bud'ko, S. L., and Canfield, P. C. (2009b). Physica C, 469: 667-673.

Prozorov, R., Tanatar, M. A., Roy, B., Ni, N., Bud'ko, S. L., and Canfield, P. C. (2010). Phys. Rev. B, 81:094509.

Prozorov, R., Tanatar, M. A., Bing Shen, Peng Cheng, Hai-Hu Wen, Bud'ko S. L., and Canfield, P. C. (2010b). Phys. Rev. B, 82:180513(R).

Putti, M., Pallecchi, I., Bellingeri, E., Cimberle, M. R., Tropeano, M., Ferdeghini, C., Palenzona, A. C., Tarantini, Yamamoto, A., Jiang, J., Jaroszynski , J., Kametani, F., Abraimov, D., Polyanskii, A., Weiss, J.D., Hellstrom, E. E., Gurevich, A., Larbalestier, D. C., Jin, R., Sales, B. C., Sefat, A. S., McGuire, M. A., Mandrus, D., Cheng, P., Jia, Y., Wen, H. H., Lee, S., Eom, C. B. (2010). Supercond. Sci. Technol., 23:034003

Rotter, M., Tegel, M., and Johrendt D. (2008). Phys. Rev. Lett., 101:107006.

Shatz, S., Shaulov, A., and Yeshurun, Y. (1993). Phys. Rev. B, 48:13871.

Shen, B., Cheng, P., Wang, Z., Fang, L., Ren, C., Shan, L., and Wen, H.-H. (2010). Phys. Rev. B, 8:014503.

Song, Y. J., Ghim, J. S., Min, B. H., Kwon, Y. S., Jung, M. H., and Rhyee, J.-S. (2010). App. Phys. Lett., 96:212508.

Tanatar, M. A., Ni, N., Martin, C., Gordon, R. T., Kim, H., Kogan, V. G., Samolyuk, G. D., Bud'ko, S. L., Canfield, P. C., and Prozorov, R. (2009). Phys. Rev. B, 
79:094507.

van der Beek, C. J., Rizza, G., Konczykowski, M., Fertey, P., Monnet, I., Klein, T., Okazaki, R., Ishikado, M., Kito, H., Iyo, A., Eisaki, H., Shamoto, S., Tillman, M. E., Bud'ko S. L., Canfield, P. C., Shibauchi, T., and Matsuda, Y. (2010). Phys. Rev. $B, 81: 174517$.

Vannette, M.D. (2008). Dynamic magnetic susceptibility of the systems with longrange magnetic order. PhD dissertation, lowa State University.

Vannette, M. D., Sefat, A. S., Jia, S., Law, S. A., Lapertot, G., Bud'ko, S. L.,

Canfield, P. C., Schmalian, J., and Prozorov, R. (2008b). J. Magn. Mat., 320:354.

Vandegrift, C.T. (1975a). Tunnel diode oscillator for 0.001 ppm measurement at low temperatures. PhD dissertation, University of Californta, Irvine.

Vandegrift, C.T. (1975b). Rev. Sci. Instrum., 46:599.

Vinokur, V. M., Feigel'man, M. V., and Geshkenbein V. B. (1991). Phys. Rev. Lett., 67:915.

Yang, H., Luo, H., Wang, Z., and Wen, H.-H. (2008a). Appl. Phys.Lett., 93:142506.

Yang, H., Ren, C., Shan, L., and Wen, H.-H. (2008b). Phys. Rev. B, 78:092504.

Yeshurun, Y., Malozemoff, A. P., and Shaulov, A. (1996). Rev. Mod. Phys., 68:911. 